\title{
Orbital and electronic entanglement in quantum teleportation schemes
}

\author{
Anna Galler $\oplus^{1}$ and Patrik Thunström $\oplus^{2}$ \\ ${ }^{1}$ Centre de Physique Théorique, Ecole Polytechnique, Institut Polytechnique de Paris, 91128 Palaiseau Cedex, France \\ ${ }^{2}$ Department of Physics and Astronomy, Materials Theory, Uppsala University, 75120 Uppsala, Sweden
}

(Received 29 November 2020; accepted 6 July 2021; published 6 August 2021)

\begin{abstract}
With progress toward more compact quantum computing architectures, fundamental questions regarding the entanglement of indistinguishable particles need to be addressed. In a solid state device, this quest is naturally connected to the quantum correlations of electrons. Here, we analyze the formation of orbital (mode) and particle entanglement in strongly correlated materials due to the Coulomb interaction between the electrons. We extend the analysis to include spectroscopic measurements of the electronic structure, with a particular focus on the photoemission process. To study the role of the different forms of electronic entanglement, including the effect of particle-number superselection rules, we propose and analyze three different electronic teleportation schemes: quantum teleportation within (i) a molecule on graphene, (ii) a nitrogen-vacancy center, and (iii) a quantum dot array.
\end{abstract}

DOI: 10.1103/PhysRevResearch.3.033120

\section{INTRODUCTION}

Entanglement lies at the heart of quantum mechanics and has been investigated extensively during the past few decades mainly due to its importance in quantum information, cryptography and teleportation [1]. The vast majority of studies focuses on the entanglement of distinguishable particles, while the entanglement of identical particles such as electrons has received much less attention so far. The experimental realizations of these quantum information processes consist, nevertheless, mainly of identical particles like photons [2,3], ultracold atoms in an optical trap [4,5], or electrons in a quantum dot $[6,7]$. The particles are instead made distinguishable by restricting their states to nonoverlapping sections of the Hilbert space, for example by a macroscopical separation of their positions. However, if one aims at building a compact quantum computer, then entanglement between identical particles in overlapping orbitals can no longer be neglected. There are in addition several outstanding questions regarding the entanglement between electrons in strongly correlated materials. For example, how is entanglement between the electrons formed within a material, and how does it affect the properties of the material? The purpose of the present study is to investigate the entanglement of electrons in a material and in electronic quantum information processes. To this aim we will propose and analyze three solid-state quantum teleportation protocols.

In contrast to the single definition of entanglement between distinguishable particles, there are two complementary

Published by the American Physical Society under the terms of the Creative Commons Attribution 4.0 International license. Further distribution of this work must maintain attribution to the author(s) and the published article's title, journal citation, and DOI. forms of entanglement for electrons; orbital (mode) entanglement [8-18] and particle entanglement [17-24]. The first form is based on a bipartition of the orbitals in the system, much like the bipartition used for distinguishable states, and quantifies the quantum correlations between the two sets of orbitals. It can be seen as a resource for sending quantum information between the orbital partitions. The definition of particle entanglement focuses instead directly on the quantum correlations between the electrons, i.e., how far the state of the system is from a statistical mixture of Fock states (single Slater determinants). As detailed in Sec. II, particle entanglement arises exclusively from particle interactions, including the interaction with a detector, while mode entanglement is generated by both nonlocal interactions and the kinetic energy term in the Hamiltonian.

The definition of mode entanglement can be supplemented with system and quantum protocol-dependent superselection rules $[13-16,25]$. In the context of quantum teleportation protocols, which transfer the state of an electron in a local orbital partition to a remote orbital partition, it is natural to impose that only operations that conserve the local particle number (N-SSR) are allowed. The N-SSR restricted mode entanglement measure of Wiseman and Vaccaro in Ref. [14] models the effect of this restriction by first measuring the local particle number of the state before its mode entanglement is measured. In the following we will therefore analyze both the standard definition of mode entanglement, and the N-SSR restricted mode entanglement.

The paper is organized as follows. An in-depth introduction of mode entanglement, particle entanglement, and N-SSR restricted mode entanglement in the second quantization formalism is provided in Appendixes A and B. In Sec. II of the main article we study of the intrinsic processes that generate mode and particle entanglement in materials. We use the derived theory to analyze how a spectroscopic measurement of the electronic structure affects the particle entanglement, with 
a particular focus on the photoemission process. In Sec. III we analyze the role of the different forms of entanglement in three different quantum teleportation schemes for electrons. We find that N-SSR restricted mode entanglement is a key resource for the quantum teleportation of electrons, in line with Refs. [26-28], but only under the condition that the teleported state is not affected by a local particle number measurement at the end of the teleportation protocol. Our work provides a new perspective to the investigation of electronic entanglement in materials and teleportation schemes (for related work see Refs. [16,26-30]), giving concrete solid-state examples and highlighting the connection between particle entanglement and N-SSR restricted mode entanglement.

\section{ENTANGLEMENT IN A MATERIAL}

\section{A. The Hamiltonian}

The evolution of the electrons within a material is governed by the Schrödinger equation and a many-body Hamiltonian composed of a one-particle term $\left(\hat{H}^{(1)}\right)$ and the two-particle Coulomb interaction $(\hat{U})$. The one-particle term $\hat{H}^{(1)}$ can in general be written

$$
\hat{H}^{(1)}=\sum_{m n} H_{m n}^{(1)} \hat{c}_{m}^{\dagger} \hat{c}_{n},
$$

where $H_{m n}^{(1)}=\left\langle 0\left|\hat{c}_{m} \hat{H}^{(1)} \hat{c}_{n}^{\dagger}\right| 0\right\rangle$ is the matrix representation of $\hat{H}^{(1)}$ evaluated in the one-particle Slater determinant basis $\hat{c}_{n}^{\dagger}|0\rangle$. The unscreened two-particle Coulomb interaction is given by

$$
\hat{U}=\sum_{\sigma \sigma^{\prime}} \iint \hat{c}_{r, \sigma}^{\dagger} \hat{c}_{r^{\prime}, \sigma^{\prime}}^{\dagger} \frac{1}{\left|r-r^{\prime}\right|} \hat{c}_{r^{\prime}, \sigma^{\prime}} \hat{c}_{r, \sigma} d r d r^{\prime},
$$

where $r$ and $\sigma$ are the position and the spin of the electron, respectively.

All materials, except solid hydrogen, have some contracted atomic-like (core) orbitals that are always completely filled with electrons due to their large attractive interaction with the nucleus. The electrons in these core orbitals can therefore be traced out of the system. The interaction term between these core electrons and the remaining (valence) electrons is then reduced to an additional effective one-particle potential term in $\hat{H}^{(1)}$. The kinetic energy term in $\hat{H}^{(1)}$ cause the remaining atomic orbitals to hybridize with the orbitals of the neighboring atoms, but the strength of the hybridization depends strongly on the overlap between the orbitals. The $3 d$ orbitals of first row transition metal atoms and the $f$ orbitals of the lanthanides and actinides are particularly contracted compared to the more extended valence $s$ - and $p$ orbitals. The weaker hybridization increase the relative importance of the $\hat{U}$ term within these contracted orbitals. The strongly hybridizing valence $s$ and $p$ orbitals are, on the other hand, often well described by mean-field-like approximations that reduce the $\hat{U}$ term to an effective potential in $\hat{H}^{(1)}$ [31-33]. Strongly correlated materials, i.e., materials that can not even qualitatively be described by only an effective $\hat{H}^{(1)}$, have therefore in general partially filled localized $d$ or $f$ orbitals.

The two terms of the Hamiltonian, $\hat{H}^{(1)}$ and $\hat{U}$, do not in general commute, but the Trotter decomposition of the resulting evolution operator

$$
e^{i \hat{H} t}=\lim _{M \rightarrow \infty}\left[e^{i \hat{H}^{(1)} t / M} e^{i \hat{U} t / M}\right]^{M},
$$

allows us to consider the effect of the one-particle unitary operator $\hat{W}^{(1)}=e^{i \hat{H}^{(1)} t / M}$ and the two-particle unitary operator $\hat{W}^{(2)}=e^{i \hat{U} t / M}$ separately.

\section{B. The one-particle evolution operator}

It is well known that the evolution given by $\hat{W}^{(1)}$ simply causes a unitary transformation of the orbitals,

$$
\hat{W}^{(1)} \hat{c}_{\mathbf{i}_{1}}^{\dagger} \hat{c}_{\mathbf{i}_{2}}^{\dagger} \cdots \hat{c}_{\mathbf{i}_{N}}^{\dagger}|0\rangle=\hat{c}_{\mathbf{i}_{1}}^{\prime \prime \dagger} \hat{c}_{\mathbf{i}_{2}}^{\prime \prime \dagger} \cdots \hat{c}_{\mathbf{i}_{N}}^{\prime \prime \dagger}|0\rangle
$$

with $\hat{c}_{n}^{\prime \prime \dagger}=\sum_{m} c_{m}^{\dagger} W_{m n}^{(1)}$. This implies, by definition, that $\hat{W}^{(1)}$ does not affect the particle entanglement in the system. The orbital transformation can nevertheless affect the mode entanglement between two orbital partitions $A$ and $B$, unless $H^{(1)}$ and thus $\hat{W}^{(1)}$ is purely local in $A$ and $B$. Local orbital transformations do not affect the mode entanglement since $\hat{\rho}_{A}$ is invariant under any local unitary orbital transformation in $B$, and $S\left[\hat{\rho}_{A}\right]$ is independent of the unitary orbital transformations in $A$. A nonzero off-diagonal element in $W^{(1)}$ between $A$ and $B$ can, on the other hand, easily affect the mode entanglement since it induces coherent transport of electrons between the two partitions. If the orbital partitions are chosen to be local and nonoverlapping in space, then it is the kinetic energy term in $\hat{H}^{(1)}$ that generate these off-diagonal element as the effective one-particle potential energy term in $\hat{H}^{(1)}$ is local in space.

In the next section we will analyze how $\hat{W}^{(2)}$ affects the mode and particle entanglement. Let us therefore, as a starting point, derive Eq. (4) for $\hat{W}^{(1)}$ and then in the next section generalize it to $\hat{W}^{(2)}$.

The unitary operator $\hat{W}^{(1)}$ can be written as

$$
\hat{W}^{(1)}=\exp \left[i \sum_{m n} \frac{H_{m n}^{(1)} t}{M} \hat{c}_{m}^{\dagger} \hat{c}_{n}\right] .
$$

The exponent can be brought to a diagonal form by diagonalizing $H^{(1)}$ using the eigenvectors $v_{m j}$ and the eigenvalues $E_{j}^{(1)}$,

$$
\hat{W}^{(1)}=\exp \left[i \sum_{j} \frac{E_{j}^{(1)} t}{M} \hat{c}_{j}^{\prime \dagger} \hat{c}_{j}^{\prime}\right]
$$

where $\hat{c}_{j}^{\prime \dagger}=\sum_{m} c_{m}^{\dagger} v_{m j}$. Since the diagonal terms in the exponent commute, and $\hat{c}_{j}^{\prime \dagger} \hat{c}_{j}^{\prime}=\hat{c}_{j}^{\prime \dagger} \hat{c}_{j}^{\prime} \hat{c}_{j}^{\prime \dagger} \hat{c}_{j}^{\prime}$, we can Taylor expand $\hat{W}^{(1)}$ as

$$
\hat{W}^{(1)}=\prod_{j}\left[1-\hat{c}_{j}^{\prime \dagger} \hat{c}_{j}^{\prime}+e^{i E_{j}^{(1)} t / M} \hat{c}_{j}^{\prime \dagger} \hat{c}_{j}^{\prime}\right] .
$$

Given Eq. (7) and that $\hat{c}_{j}^{\prime \dagger} \hat{c}_{j}^{\prime \dagger}=0$, it follows that

$$
\hat{W}^{(1)} \hat{c}_{j}^{\dagger \dagger}=\hat{c}_{j}^{\prime \dagger} e^{\frac{i E_{j}^{(1)} t}{M}} \hat{W}^{(1)} .
$$


Hence, if $\hat{W}^{(1)}$ acts on a creation operator $\hat{c}_{n}^{\dagger}=\sum_{j} c_{j}^{\prime \dagger} v_{n j}^{*}$ from the left, then we get

$$
\begin{aligned}
\hat{W}^{(1)} \hat{c}_{n}^{\dagger} & =\hat{W}^{(1)} \sum_{j} c_{j}^{\prime \dagger} v_{n j}^{*} \\
& =\left[\sum_{j} \hat{c}_{j}^{\dagger \dagger} e^{i E_{j}^{(1)} t / M} v_{n j}^{*}\right] \hat{W}^{(1)} \\
& =\sum_{j k m} \hat{c}_{k}^{\prime \dagger} v_{m k}^{*} v_{m j} e^{i E_{j}^{(1)} t / M} v_{n j}^{*} \hat{W}^{(1)} \\
& =\sum_{m} \hat{c}_{m}^{\dagger} W_{m n}^{(1)} \hat{W}^{(1)},
\end{aligned}
$$

where we in the third line used the Kronecker delta $\delta_{k j}=$ $\sum_{m} v_{m k}^{*} v_{m j}$ and that

$$
W_{m n}^{(1)}=\left(e^{i H^{(1)} t / M}\right)_{m n}=\sum_{j} v_{m j} e^{i E_{j}^{(1)} t / M} v_{n j}^{*} .
$$

Equation (4) follows immediately from Eq. (9) and that $\hat{W}^{(1)}|0\rangle=|0\rangle$.

\section{The two-particle evolution operator}

The Coulomb interaction $\hat{U}$ in Eq. (2) is diagonal in the position and spin basis $\left(r, \sigma, r^{\prime}, \sigma^{\prime}\right)$. We can therefore also write $\hat{W}^{(2)}$ in a diagonal form, cf. Eq. (6),

$$
\begin{aligned}
\hat{W}^{(2)} & =\exp \left[\frac{i t}{M} \sum_{\sigma \sigma^{\prime}} \iint \frac{t / N}{\left|r-r^{\prime}\right|} \hat{c}_{r \sigma}^{\dagger} \hat{c}_{r^{\prime} \sigma^{\prime}}^{\dagger} \hat{c}_{r^{\prime} \sigma^{\prime}}, \hat{c}_{r \sigma} d r^{\prime} d r\right], \\
& \equiv \exp \left[i \sum_{\mathbf{r} \in \mathcal{S}_{2}} \frac{E_{\mathbf{r}} t}{M} \hat{S}_{\mathbf{r}}^{\dagger} \hat{S}_{\mathbf{r}}\right],
\end{aligned}
$$

where the two-particle Slater determinant index $\mathbf{r}$ contains both position and spin, i.e., $\hat{S}_{\mathbf{r}}=\hat{c}_{r \sigma} \hat{c}_{r^{\prime} \sigma^{\prime}}$. The Taylor expansion of $\hat{W}^{(2)}$ becomes

$$
\hat{W}^{(2)}=\prod_{\mathbf{r} \in \mathcal{S}_{2}}\left(1-\hat{S}_{\mathbf{r}}^{\dagger} \hat{S}_{\mathbf{r}}+e^{\frac{i E_{\mathbf{r}}}{M}} \hat{S}_{\mathbf{r}}^{\dagger} \hat{S}_{\mathbf{r}}\right),
$$

which after some algebra yields

$$
\begin{aligned}
\hat{W}^{(2)} \hat{c}_{r \sigma}^{\dagger} & =\hat{c}_{r \sigma}^{\dagger} \prod_{\left(r \sigma r^{\prime} \sigma^{\prime}\right) \in \mathcal{S}_{2}}\left[1-\hat{c}_{r^{\prime} \sigma^{\prime}}^{\dagger} \hat{c}_{r^{\prime} \sigma^{\prime}}+e^{\frac{i t\left(r \sigma r r^{\prime} \sigma^{\prime}\right)}{M}} \hat{c}_{r^{\prime} \sigma^{\prime}}^{\dagger} \hat{c}_{r^{\prime} \sigma^{\prime}}\right] \hat{W}^{(2)} \\
& =\hat{c}_{r \sigma}^{\dagger} \exp \left[i t / M \sum_{\left(r \sigma r^{\prime} \sigma^{\prime}\right) \in \mathcal{S}_{2}} E_{\left(r \sigma r^{\prime} \sigma^{\prime}\right)} \hat{c}_{r^{\prime} \sigma^{\prime}}^{\dagger} \hat{c}_{r^{\prime} \sigma^{\prime}}\right] \hat{W}^{(2)} \\
& \equiv \hat{c}_{r \sigma}^{\dagger} \hat{W}_{r \sigma}^{(1)} \hat{W}^{(2)},
\end{aligned}
$$

where $\left(r \sigma r^{\prime} \sigma^{\prime}\right)$ denotes the Slater determinant index for which the electrons occupy $r$ and $r^{\prime}$ with spin $\sigma$ and $\sigma^{\prime}$, respectively. $\hat{W}_{r \sigma}^{(1)}$ is a one-particle unitary operator that describes the Coulomb potential due to the electron at position $r$. It has the same form as $\hat{W}^{(1)}$ in Eq. (6) except that it also depends on the position and spin of the creation operator $\hat{W}^{(2)}$ acted on. It is straightforward to generalize Eq. (13) to treat an effective $N$-body density-density interaction by replacing $r^{\prime}$ and $\hat{c}_{r^{\prime}}^{\dagger} \hat{c}_{r^{\prime}}$ with an $N-1$ electron Slater determinant index $\mathbf{r}^{\prime}$ and the corresponding product of creation and annihilation operators $\hat{S}_{\mathbf{r}^{\prime}}^{\dagger} \hat{S}_{\mathbf{r}^{\prime}}$, respectively.
The main difference between Eq. (8) and Eq. (13) is that the unitary operator $\hat{W}_{r \sigma}^{(1)}$ can not be absorbed by an orbital transformation. Instead, $\hat{W}_{r \sigma}^{(1)}$ will act on the next creation operator $\hat{c}_{r^{\prime} \sigma^{\prime}}^{\dagger}$ in line giving

$$
\hat{W}_{r \sigma}^{(1)} \hat{c}_{r^{\prime} \sigma^{\prime}}^{\dagger}=\hat{c}_{r^{\prime} \sigma^{\prime}}^{\dagger}, e^{i E_{\left(r r^{\prime} \sigma^{\prime}\right)} t / M} \hat{W}_{r \sigma}^{(1)} .
$$

Equations (13) and (14) fully determine the evolution given by $\hat{W}^{(2)}$,

$$
\begin{aligned}
\hat{W}^{(2)} \hat{c}_{\mathbf{r}_{1}}^{\dagger} \hat{c}_{\mathbf{r}_{2}}^{\dagger} \cdots \hat{c}_{\mathbf{r}_{N}}^{\dagger}|0\rangle & =\hat{c}_{\mathbf{r}_{1}}^{\dagger} \hat{W}_{\mathbf{r}_{1}}^{(1)} \hat{c}_{\mathbf{r}_{2}}^{\dagger} \hat{W}_{\mathbf{r}_{2}}^{(1)} \cdots \hat{c}_{\mathbf{r}_{N}}^{\dagger}|0\rangle \\
& =\prod_{j=1}^{N} \hat{c}_{\mathbf{r}_{j}}^{\dagger} e^{\frac{i t}{2 M} \sum_{n \neq j}^{N} E_{\left(r_{n} r_{i}\right)}}|0\rangle .
\end{aligned}
$$

The phase factors induced by $\hat{W}^{(2)}$ in the second line of Eq. (15) depend nonlinearly on the orbital occupations. It is therefore not possible to assign to each orbital a fixed phase shift as in Eq. (8), unless the many-body state is an eigenstate of $\hat{U}$. Hence, in contrast to one-particle term $\hat{H}^{(1)}$ in the Hamiltonian, the evolution given by the Coulomb interaction will in general modify the particle entanglement. The mode entanglement between two orbital partitions $A$ and $B$ is also in general affected by $\hat{W}^{(2)}$. However, just as for $\hat{W}^{(1)}$, it requires a state-dependent shift of the relative phases between the electrons in $A$ and $B$ in Eq. (15), i.e., that the electrons in $A$ and $B$ interact with each other.

These effects can be illustrated with a minimal model; a system with two orbitals 1 and 2 and two electrons, with $H^{(1)}=0$ and an on-site effective Coulomb interaction of the form

$$
\hat{U}=U \hat{c}_{1 \uparrow}^{\dagger} \hat{c}_{1 \downarrow}^{\dagger} \hat{c}_{1, \downarrow} \hat{c}_{1 \uparrow}+U \hat{c}_{2 \uparrow}^{\dagger} \hat{c}_{2 \downarrow}^{\dagger} \hat{c}_{2 \downarrow} \hat{c}_{2 \uparrow} .
$$

The two electrons are prepared in the Fock state $|\psi\rangle=$ $\hat{c}_{a \uparrow}^{\dagger} \hat{c}_{b \downarrow}^{\dagger}|0\rangle$ where

$$
\hat{c}_{a \sigma}^{\dagger}=\frac{1}{\sqrt{2}}\left(\hat{c}_{1 \sigma}^{\dagger}+\hat{c}_{2 \sigma}^{\dagger}\right) \quad \hat{c}_{b \sigma}^{\dagger}=\frac{1}{\sqrt{2}}\left(\hat{c}_{1 \sigma}^{\dagger}-\hat{c}_{2 \sigma}^{\dagger}\right) .
$$

The state $|\psi\rangle$ evolve according to Eq. (15),

$$
\begin{aligned}
W^{(2)} & |\psi\rangle \\
= & \frac{W^{(2)}}{2}\left(\hat{c}_{1 \uparrow}^{\dagger} \hat{c}_{1 \downarrow}^{\dagger}-\hat{c}_{1 \uparrow}^{\dagger} \hat{c}_{2 \downarrow}^{\dagger}+\hat{c}_{2 \uparrow}^{\dagger} \hat{c}_{1 \downarrow}^{\dagger}-\hat{c}_{2 \uparrow}^{\dagger} \hat{c}_{2 \downarrow}^{\dagger}\right)|0\rangle \\
= & \frac{1}{2}\left(e^{i U t} \hat{c}_{1 \uparrow}^{\dagger} \hat{c}_{1 \downarrow}^{\dagger}-\hat{c}_{1 \uparrow}^{\dagger} \hat{c}_{2 \downarrow}^{\dagger}+\hat{c}_{2 \uparrow}^{\dagger} \hat{c}_{1 \downarrow}^{\dagger}-e^{i U t} \hat{c}_{2 \uparrow}^{\dagger} \hat{c}_{2 \downarrow}^{\dagger}\right)|0\rangle \\
= & \left(\frac{e^{i U t}+1}{2} \hat{c}_{a \uparrow}^{\dagger} \hat{c}_{b \downarrow}^{\dagger}+\frac{e^{i U t}-1}{2} \hat{c}_{a \downarrow}^{\dagger} \hat{c}_{b \uparrow}^{\dagger}\right)|0\rangle .
\end{aligned}
$$

As $|\psi\rangle$ evolves it goes from a Fock state at $t=0$ to become a maximally particle entangled state at $t=\frac{\pi}{2 U}$. The mode entanglement between orbitals $a$ and $b$ changes at the same time from $S\left[\rho_{a}(t=0)\right]=0$ to $S\left[\rho_{a}\left(t=\frac{\pi}{2 U}\right)\right]=1 / 2$. The mode entanglement between 1 and 2 remains, on the other hand, constant at $S=3 / 4$ since the on-site effective Coulomb interaction is purely local with respect to 1 and 2 .

\section{Particle entanglement and measurements}

The particle entanglement is not only affected by the interactions within a system but also by the interaction with a 
measurement device. The underlying principle of any measurement device is a nonlinear amplification process that is triggered by its interaction with the probed system. The amplification process is in general a cascade reaction designed to correlate the states of a macroscopically large number of particles with the state of the probed system. For example, an electron multiplier measures the occupation of a free electron orbital (e) at time $t=0$ by accelerating the (primary) electron toward a surface of a secondary-emissive material. The interaction with the surface electrons $\left(D^{0}\right)$ cause the ejection of secondary electrons which in turn are accelerated toward a second surface where the process is repeated. The chain reaction cause a cascade of excited electrons $\left(D^{1}\right)$ to travel down the electron multiplier to finally, at time $t=T$, reach an electric readout. An electron multiplier will in general detect the presence of electrons in more than a single spin-orbital. This can, however, be treated as an array of ideal detectors each detecting the occupation of just a single spin-orbital but having a common electrical readout. The ideal one-particle detection process of an electron in orbital $e$ can hence be summarized as

$$
\begin{array}{r}
\left|\Psi^{i}(t=0)\right\rangle=\hat{c}_{e}^{\dagger}\left[\sum_{\mathbf{i} \in \mathcal{S}_{N}} D_{\mathbf{i}}^{0}(0) \hat{S}_{\mathbf{i}}^{\dagger}\right]|0\rangle \\
\rightarrow\left|\Psi^{f}(t=T)\right\rangle=\left[\sum_{\mathbf{i} \in \mathcal{S}_{N+1}} D_{\mathbf{i}}^{1}(T) \hat{S}_{\mathbf{i}}^{\dagger}\right]|0\rangle .
\end{array}
$$

The pointer states of the detector $\left(D^{0}\right.$ and $\left.D^{1}\right)$ are at equal time $t=T$ for all practical purposes irreversibly orthogonal to each other, i.e.,

$$
\left\langle\Psi^{i}(t=T)\left|\hat{W}\left(t^{\prime}\right)\right| \Psi^{f}(t=T)\right\rangle=0,
$$

where $\hat{W}\left(t^{\prime}\right)$ is any practically achievable evolution operator, and the time $t^{\prime}$ can be made arbitrarily large by increasing the size of the detector. To highlight this irreversibility we will in the following use a notation that separates the state of the probed system $(\psi)$ and the detector $(D)$,

$$
|\Psi(t)\rangle=\left|\psi(t), D^{p}(t)\right\rangle \equiv\left[\sum_{\mathbf{i}} \psi_{\mathbf{i}}(t) \hat{S}_{\mathbf{i}}^{\dagger}\right]\left[\sum_{\mathbf{j}} D_{\mathbf{j}}^{p}(t) \hat{S}_{\mathbf{j}}^{\dagger}\right]|0\rangle .
$$

The interaction between the system and the detector in Eq. (19) causes in general the formation of a superposition between the different pointer states,

$$
\begin{aligned}
\left|\Psi^{i}\right\rangle & \equiv \sqrt{\alpha} e^{i \theta} \hat{c}_{e}^{\dagger}\left|\psi_{e}, D^{0}(0)\right\rangle+\sqrt{1-\alpha}\left|\psi_{s}, D^{0}(0)\right\rangle \\
\rightarrow\left|\Psi^{f}\right\rangle & \equiv \sqrt{\alpha} e^{i \theta}\left|\psi_{e}, D^{1}(T)\right\rangle+\sqrt{1-\alpha}\left|\psi_{s}, D^{0}(T)\right\rangle,
\end{aligned}
$$

where $0 \leqslant \alpha \leqslant 1$ and the state $\left|\psi_{s}\right\rangle$ does not trigger the detector. However, since the different pointer states of the detector always remain orthogonal to each other it is impossible to access the relative phase $e^{i \theta}$ in $\left|\psi^{f}\right\rangle$. The irreversible lack of interference makes the superposition between the different pointer states equivalent to a statistical (classical) correlation. The particle entanglement within the detector, i.e., $E\left[\left|0, D^{0}\right\rangle\right]$ and $E\left[\left|0, D^{1}\right\rangle\right]$ (see Appendix A), is in general equally unaccessible. In the following we will therefore focus on the particle entanglement within the probed system $(S)$ given by
$E\left[\hat{P}_{D^{p}}\left|\Psi^{f}\right\rangle\right]$, where the projection operator

$$
\hat{P}_{D^{p}} \equiv \sum_{\substack{\mathbf{i} \in \mathcal{S}^{D} \\ \mathbf{j} \in \mathcal{S}^{S}}} D_{\mathbf{i}}^{p *} \hat{P}_{\mathbf{j i}}
$$

resolves and projects out the pointer state $D^{p}$ of the detector using the projectors $\hat{P}_{\mathrm{ji}}$ defined by Eq. (A12).

As shown in Appendix D, if the normalized states $\left|\psi_{e}\right\rangle$ and $\left|\psi_{s}\right\rangle$ contain $N$ and $N+1$ electrons, respectively, but neither state has an electron in orbital $e$, then the particle entanglement fulfills the inequality

$$
\begin{aligned}
& E\left[\sqrt{\alpha} e^{i \theta} \hat{c}_{e}^{\dagger}\left|\psi_{e}\right\rangle+\sqrt{1-\alpha}\left|\psi_{s}\right\rangle\right] \\
& \quad \geqslant \alpha E\left[\left|\psi_{e}\right\rangle\right]+(1-\alpha) E\left[\left|\psi_{s}\right\rangle\right],
\end{aligned}
$$

for both $E_{G}[|\psi\rangle]$ and $S[|\psi\rangle]$. Equation (24) can be rewritten as

$$
E\left[\hat{P}_{D^{0}}\left|\psi^{i}\right\rangle\right] \geqslant E\left[\hat{P}_{D^{1}}\left|\psi^{f}\right\rangle\right]+E\left[\hat{P}_{D^{0}}\left|\psi^{f}\right\rangle\right],
$$

for the states in Eq. (22), which shows that the measurement of the occupation of a single spin-orbital does not increase the particle entanglement of the probed system on average. This parallels how local measurements and classical communication do not increase the entanglement of distinguishable particles on average [34].

The interaction with the detector is often preceded by an excitation of the probed system. In photoemission spectroscopy the system is first excited by a photon which causes an electron to be emitted from the surface of the sample. The electron enters a highly excited free electron orbital and is then captured by an electron multiplier as in Eq. (22). If the excitation by the photon is considered to be much faster than the dynamics of the system, then it can be described by an effective one-particle unitary operator $\left(\hat{W}^{(1)}\right)$. This implies that the full ideal photoemission spectroscopy measurement also fulfills the particle entanglement inequality in Eq. (25) and not only the detection step, i.e., a photoemission measurement does not increase the particle entanglement.

The working principle of a photomultiplier is the same as an electron multiplier except that the primary particle is a photon instead of an electron. The incoming photon hits a photoemissive surface which in the ideal case cause the emission of a free electron. The free electron is then detected by an electron multiplier according to Eq. (22). Although the detection of the free electron fulfills the particle entanglement inequality in Eq. (25), the detection of a photon emitted by the system may still cause the formation of particle entanglement within the system. The reason is that the combined emission and absorption of a photon corresponds to a Coulomb interaction which may cause particle entanglement to form within the probed system according to Eq. (15).

The particle number projection in the definition of the $\mathrm{N}$ SSR restricted mode entanglement corresponds to a projective quantum nondemolition measurement $[35,36]$ in which the total number of electrons in an orbital partition is probed instead of the occupation of individual spin-orbitals. This can in general only be achieved by letting the electrons in the probed system interact with an auxiliary system, and then measure the state of the auxiliary system. The interaction between the two systems can increase the average particle entanglement of 


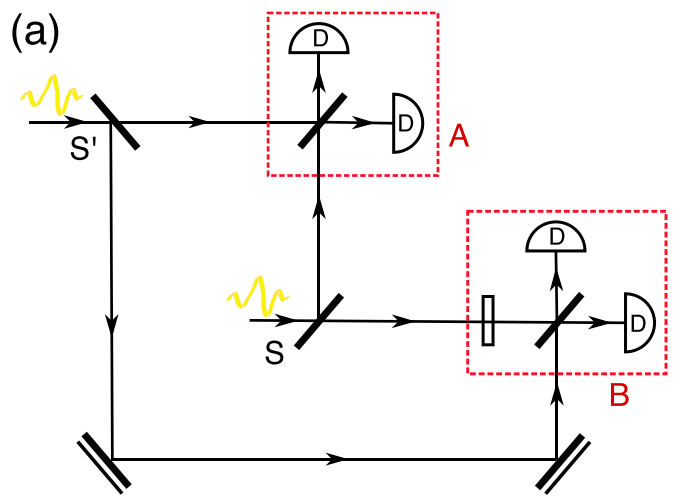

(b)

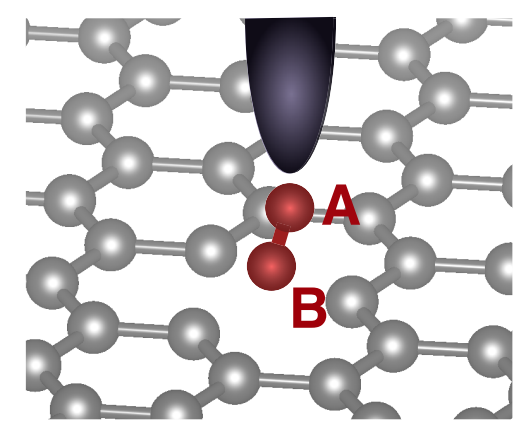

FIG. 1. (a) Photonic and (b) electronic teleportation schemes. (a) In the photon teleportation scheme of Ref. [37] an entangled resource state shared between $A($ lice $)$ and $B(o b)$ is created through a single photon entering a 50/50 beam-splitter $S$. The state to be teleported instead is generated at $S^{\prime}$. A(lice) performs a projective measurement on her part of the resource state and the state to be teleported ( $D$ are detectors). Depending on the outcome of this measurement, $B(o b)$ needs to perform a suitable operation (phase shift) on his state in order to obtain the teleported state. b) In our electron teleportation scheme the two $\mathrm{H}$ atoms of an $\mathrm{H}_{2}$ molecule play the role of $A($ lice $)$ and $B(o b)$. The $\mathrm{H}_{2}$ molecule is adsorbed on a single vacancy in graphene and can be addressed by a spin-polarized STM-tip. The latter can be used to add an electron to the molecule [state to be teleported, see Eq. (28)] and to perform a magnetization measurement.

the probed system, which is the physical reason why a mode entangled Fock state can become particle entangled after the projective occupation measurement.

\section{FERMIONIC TELEPORTATION}

In this section we propose and analyze three different electronic teleportation schemes: quantum teleportation within a molecule on graphene (Sec. III A), a nitrogen-vacancy center (Sec. III B), and a quantum dot array (Sec. III C) to study the roles of the different forms of electronic entanglement.

\section{A. Electron teleportation in a hydrogen molecule}

Our first teleportation scheme can be seen as a molecular analog of the photon teleportation scheme presented in Ref. [37] which is schematically depicted in Fig. 1(a). In this example we consider a hydrogen molecule that is adsorbed on graphene and stabilized by a magnetic scanning tunneling microscope (STM) tip, as shown in Fig. 1(b). The hydrogen molecule is adsorbed on a single vacancy site since this adsorption site is favored by its binding energy $(\approx 0.4 \mathrm{eV})$ [38]. An experimental realization of this scheme could be facilitated by using a larger, even more stable molecule, as long as the orbital structure is similar.

In Fig. 1(b) the two constituting hydrogen atoms $\mathrm{H}$ of the molecule are labeled $A$ and $B$, respectively, and will play the role of Alice and Bob. We assume that the molecule is initially in its ionized state $\mathrm{H}_{2}^{+}$, with one spin $\downarrow$ electron in its binding orbital $\sigma$ (with the spin pointing in the in-plane $x$ direction). Thus, the initial wave function of the molecule reads

$$
\hat{c}_{\sigma \downarrow}^{\dagger}|0\rangle=\frac{1}{\sqrt{2}}\left(\hat{c}_{A \downarrow}^{\dagger}+\hat{c}_{B \downarrow}^{\dagger}\right)|0\rangle,
$$

where $\hat{c}_{\sigma \downarrow}^{\dagger}\left(\hat{c}_{A \downarrow}^{\dagger}\right.$ and $\left.\hat{c}_{B \downarrow}^{\dagger}\right)$ creates an electron with spin $\downarrow$ in the binding orbital $\sigma$ which is a superposition of the $s$ orbitals of $\mathrm{H}$ atoms $A$ and $B . \hat{c}_{\sigma \downarrow}^{\dagger}|0\rangle$ represents the initial resource state shared between $A($ lice $)$ and $B(o b)$. Its mode entanglement, given the bipartition between $A$ and $B$, can be calculated from the reduced density matrix $\rho_{A}$, defined in Eq. (B1). The matrix $\rho_{A}$, written in the basis $\left\{|0\rangle, \hat{c}_{A \downarrow}^{\dagger}|0\rangle, \hat{c}_{A \uparrow}^{\dagger}|0\rangle, \hat{c}_{A \downarrow}^{\dagger} \hat{c}_{A \uparrow}^{\dagger}|0\rangle\right\}$, reads

$$
\rho_{A}=\frac{1}{2}\left(\begin{array}{llll}
1 & 0 & 0 & 0 \\
0 & 1 & 0 & 0 \\
0 & 0 & 0 & 0 \\
0 & 0 & 0 & 0
\end{array}\right),
$$

which gives the mode entanglement $S\left[\rho_{A}\right]=\operatorname{Tr}\left[\rho_{A}(1-\right.$ $\left.\left.\rho_{A}\right)\right]=1 / 2$. Clearly, $\hat{c}_{\sigma \downarrow}^{\dagger}|0\rangle$ is not particle entangled nor $\mathrm{N}$ SSR mode entangled as it only contains a single electron.

Next, we inject a second electron with spin $\uparrow$ into the $\mathrm{H}_{2}^{+}$ molecule via the spin-polarized STM tip. The energy of this injected electron is chosen so that it occupies a superposition $\gamma$ of the binding $\sigma$ and antibinding $\bar{\sigma}$ orbital of the $\mathrm{H}_{2}$ molecule, giving the initial state

$$
\hat{c}_{\gamma \uparrow}^{\dagger}|0\rangle=\left[\frac{a+b}{\sqrt{2}} \hat{c}_{\sigma \uparrow}^{\dagger}+\frac{a-b}{\sqrt{2}} \hat{c}_{\bar{\sigma} \uparrow}^{\dagger}\right]|0\rangle=\left(a \hat{c}_{A \uparrow}^{\dagger}+b \hat{c}_{B \uparrow}^{\dagger}\right)|0\rangle,
$$

where $a$ and $b$ are unknown coefficients, and $|a|^{2}+|b|^{2}=1$. The (partial) state $a \hat{c}_{A \uparrow}^{\dagger}|0\rangle$ corresponds to the unknown state to be teleported from Alice's spin-orbital $A \uparrow$ to $\{\backslash \mathrm{rm}$ Bob $\} \mathrm{s}$ spin-orbital $B \downarrow$ giving the final state $\left(a \hat{c}_{B \downarrow}^{\dagger}+b \hat{c}_{B \uparrow}^{\dagger}\right)|0\rangle$. Note that in the related photonic scheme, depicted in Fig. 1(a), the state to be teleported represents a superposition between a single photon and the vacuum. However, the parity superselection rule (P-SSR) for electrons does not allow for such a superposition of even and odd particle numbers. Thus, the second arm of the interferometer in Fig. 1(a), ranging from $S^{\prime}$ to $\mathrm{B}$, which in the photonic scheme serves solely as a way to verify a successful teleportation, is an essential constituent in the electronic scheme. This extra spin-orbital $(B \uparrow)$ introduces an ambiguity in the bipartitioning of the system. If $B \uparrow$ is considered to belong to Bob, then the initial state in Eq. (28) is mode entangled with respect to $\mathrm{A}($ lice $)$ and $\mathrm{B}(\mathrm{ob})$ but the final state is not. The opposite is true if $B \uparrow$ is instead assigned to Alice. In the following analysis of the mode entanglement 
we use the former choice of the bipartition, but return to the latter choice and its impact on the N-SSR restricted mode entanglement in the end of the section.

The mode entanglement of Eq. (28) can be calculated from its reduced density matrix $\rho_{A}$

$$
\rho_{A}=\left(\begin{array}{cccc}
|b|^{2} & 0 & 0 & 0 \\
0 & 0 & 0 & 0 \\
0 & 0 & |a|^{2} & 0 \\
0 & 0 & 0 & 0
\end{array}\right)
$$

and yields $S\left[\rho_{A}\right]=1-|a|^{4}-|b|^{4} . \hat{c}_{\gamma \uparrow}^{\dagger}|0\rangle$ is again not particle entangled nor N-SSR mode entangled since it only contains a single electron.

The total state of the $\mathrm{H}_{2}$ molecule is given by

$$
|\psi\rangle \equiv \hat{c}_{\sigma \downarrow}^{\dagger} \hat{c}_{\gamma \uparrow}^{\dagger}|0\rangle=\frac{1}{\sqrt{2}}\left(\hat{c}_{A \downarrow}^{\dagger}+\hat{c}_{B \downarrow}^{\dagger}\right)\left(a \hat{c}_{A \uparrow}^{\dagger}+b \hat{c}_{B \uparrow}^{\dagger}\right)|0\rangle .
$$

$|\psi\rangle$ is mode entangled if we consider a bipartition between hydrogen atoms $A$ and $B$. In this case, the reduced density matrix $\rho_{A}$ becomes

$$
\rho_{A}=\frac{1}{2}\left(\begin{array}{cccc}
|b|^{2} & 0 & 0 & 0 \\
0 & |b|^{2} & 0 & 0 \\
0 & 0 & |a|^{2} & 0 \\
0 & 0 & 0 & |a|^{2}
\end{array}\right),
$$

which yields the entropic mode entanglement $S\left[\rho_{A}\right]=1-$ $1 / 2\left(|a|^{4}+|b|^{4}\right)$. Note that this is not simply the sum of the entropic entanglement of the states in Eqs. (26) and (28) since we are considering linear and not logarithmic entropy. $|\psi\rangle=$ $\hat{c}_{\sigma \downarrow}^{\dagger} \hat{c}_{\gamma \uparrow}^{\dagger}|0\rangle$ is not a particle-entangled state as it clearly can be written as a single Slater determinant. The calculation of the $\mathrm{N}-\mathrm{SSR}$ restricted mode entanglement of $|\psi\rangle$ requires a few more steps. The initial projective occupation measurement in its definition produce the (unnormalized) states

$$
\begin{gathered}
\left|\psi^{(0)}\right\rangle=\frac{1}{2}|0\rangle, \\
\frac{1}{\sqrt{2}}\left|\psi^{(1)}\right\rangle=\left[\frac{b}{\sqrt{2}} \hat{c}_{A \downarrow}^{\dagger} \hat{c}_{B \uparrow}^{\dagger}+\frac{a}{\sqrt{2}} \hat{c}_{B \downarrow}^{\dagger} \hat{c}_{A \uparrow}^{\dagger}\right]|0\rangle, \\
\left|\psi^{(2)}\right\rangle=\frac{1}{2} \hat{c}_{A \downarrow}^{\dagger} \hat{c}_{A \uparrow}^{\dagger}|0\rangle .
\end{gathered}
$$

The local Fock states $\left|\psi^{(0)}\right\rangle$ and $\left|\psi^{(2)}\right\rangle$ do not contribute to the mode entanglement, but the particle entangled state $\left|\psi^{(1)}\right\rangle$ $\left(E_{G}\left[1 / \sqrt{2}\left|\psi^{(1)}\right\rangle\right]=1 / 2-1 / 2 \max \left(|a|^{2},|b|^{2}\right)\right.$ does. The reduced density matrix of $1 / \sqrt{2}\left|\psi^{(1)}\right\rangle$ reads

$$
\rho_{A}=\frac{1}{2}\left(\begin{array}{cccc}
0 & 0 & 0 & 0 \\
0 & |b|^{2} & 0 & 0 \\
0 & 0 & |a|^{2} & 0 \\
0 & 0 & 0 & 0
\end{array}\right),
$$

which yields the N-SSR restricted mode entanglement

$$
S^{N}(|\psi\rangle)=\frac{1}{2}\left(1-|a|^{4}-|b|^{4}\right) .
$$

It is the projective occupation measurement of the Fock state $|\psi\rangle$ that produces the particle entanglement in the state $\left|\psi^{(1)}\right\rangle$. The presence of particle entanglement after the projective occupation measurement is indeed necessary (although not sufficient) for a state to be N-SSR mode entangled, as shown in Appendix $\mathrm{C}$, since the projection also removes any contribution from nonlocal orbitals to the mode entanglement.

The next step in our teleportation scheme is a measurement of the spin of the electrons in atom $A$ along the $y$ axis. Even if very challenging, this measurement can in principle be performed by exciting the electrons in $A$ with a photon to make them tunnel to the magnetized STM-tip which detects their spin. This causes the build up of particle entanglement between the system and the detector, but it does not increase the particle entanglement within the probed system itself as the spin measurement corresponds to two separate spin-orbital occupation measurements which both fulfill Eq. (25).

To analyze the possible outcomes of the measurement, we substitute $\hat{c}_{A \uparrow(\downarrow)}^{\dagger}=1 / \sqrt{2}\left(\hat{c}_{A \uparrow y\left({ }^{+}\right)}^{\dagger} \hat{c}_{A \downarrow y}^{\dagger}\right)$ and rewrite the initial state $|\psi\rangle$ as

$$
\begin{aligned}
|\psi\rangle= & \frac{1}{\sqrt{2}}\left[\frac{1}{\sqrt{2}}\left(\hat{c}_{A \uparrow y}^{\dagger}-\hat{c}_{A \downarrow y}^{\dagger}\right)+\hat{c}_{B \downarrow}^{\dagger}\right] \\
& \times\left[\frac{a}{\sqrt{2}}\left(\hat{c}_{A \uparrow y}^{\dagger}+\hat{c}_{A \downarrow y}^{\dagger}\right)+b \hat{c}_{B \uparrow}^{\dagger}\right]|0\rangle \\
= & \frac{1}{\sqrt{2}}\left[\frac{\hat{c}_{A \uparrow y}^{\dagger}}{\sqrt{2}}\left(-a \hat{c}_{B \downarrow}^{\dagger}+b \hat{c}_{B \uparrow}^{\dagger}\right)-\frac{\hat{c}_{A \downarrow y}^{\dagger}}{\sqrt{2}}\left(a \hat{c}_{B \downarrow}^{\dagger}+b \hat{c}_{B \uparrow}^{\dagger}\right)\right. \\
& \left.+a \hat{c}_{A \uparrow y}^{\dagger} \hat{c}_{A \downarrow y}^{\dagger}+b \hat{c}_{B \downarrow}^{\dagger} \hat{c}_{B \uparrow}^{\dagger}\right]|0\rangle .
\end{aligned}
$$

If Alice either detects no electrons (due to $\frac{b}{\sqrt{2}} \hat{c}_{B \downarrow}^{\dagger} \hat{c}_{B \uparrow}^{\dagger}|0\rangle$ ) or two electrons (due to $\frac{a}{\sqrt{2}} \hat{c}_{A \uparrow y}^{\dagger} \hat{c}_{A \downarrow y}^{\dagger}|0\rangle$ ) from atom $A$, then no teleportation can be achieved, as Bob's spin-orbitals are then either completely filled or empty, respectively, due to particle number conservation. Thus, the probability of a successful teleportation is limited to $50 \%$. The reduced success rate is reflected in the N-SSR restricted mode entanglement as it projects the state on exactly these subspaces before the mode entanglement is measured.

The two successful magnetization measurement outcomes of $|\psi\rangle$ are $\frac{1}{2} \hat{c}_{A \uparrow y}^{\dagger}\left(-a \hat{c}_{B \downarrow}^{\dagger}+b \hat{c}_{B \uparrow}^{\dagger}\right)|0\rangle$ and $\frac{1}{2} \hat{c}_{A \downarrow y}^{\dagger}\left(a \hat{c}_{B \downarrow}^{\dagger}+\right.$ $\left.b \hat{c}_{B \uparrow}^{\dagger}\right)|0\rangle$. In the latter case the electron on atom $B$ is already in the state to be teleported [see Eq. (28)], and no further operation is necessary to complete the teleportation scheme. In the former case Bob still needs to apply a "phase shift" to the electron spin on atom $B$ in order to obtain the desired outcome. This can be achieved by applying a magnetic field on atom $B$ in $\downarrow x$ direction for a specific time $t=\pi /\left(\mu B_{x}\right)$, which yields

$$
\begin{aligned}
e^{-\frac{i}{\hbar} \mu B_{x} \hat{S}_{x} t}\left(a \hat{c}_{B \downarrow}^{\dagger}-b \hat{c}_{B \uparrow}^{\dagger}\right)|0\rangle & =\left(e^{-i \frac{\pi}{2}} a \hat{c}_{B \downarrow}^{\dagger}-e^{i \frac{\pi}{2}} b \hat{c}_{B \uparrow}^{\dagger}\right)|0\rangle \\
& =-i\left(a \hat{c}_{B \downarrow}^{\dagger}+b \hat{c}_{B \uparrow}^{\dagger}\right)|0\rangle .
\end{aligned}
$$

This example shows that it is possible to teleport a state without creating or destroying particle entanglement within the system. The caveat is that there is only a $50 \%$ probability of success, which is reflected in the reduction of the mode entanglement through the particle-number superselection rule (N-SSR). A $100 \%$ success rate requires the use of Bell 


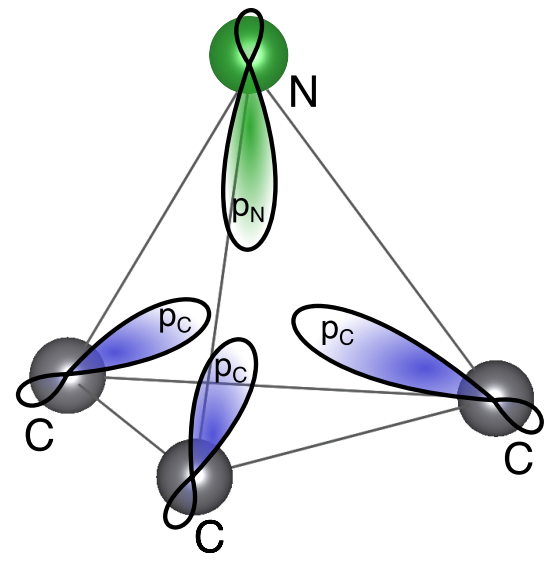

FIG. 2. Schematic visualization of a NV center in diamond and its dangling $p$ orbitals oriented toward the vacancy (in the center of the pyramid). The $\mathrm{N}-p$ orbital is labeled with $p_{N}$, while the corresponding C- $p$ orbitals are labeled with $p_{C}$.

states [39], which are maximally particle and mode entangled by construction, as illustrated in the next two examples.

In the above analysis of the mode entanglement the spinorbital $B \uparrow$ was assigned to Bob. If $B \uparrow$ is instead assigned to Alice, then the entropic mode entanglement of the total state Eq. (30) is $1 / 2$ and the N-SSR restricted mode entanglement is zero. The optimal success rate of the teleportation is nevertheless still 50\% since the teleportation protocol can remain unaltered, except for a trivial modification of the phase shift operator such that it only acts on $B \downarrow$. This illustrates that the $\mathrm{N}$-SSR restricted mode entanglement is only a useful measure of the optimal success rate if the quantum teleportation can be verified by a local measurement. This is not the case when $B \uparrow$ belongs to Alice since this makes the final teleported state mode entangled. Put differently, it is the projective local number measurement in the definition of the N-SSR restricted mode entanglement that brings the (predicted) success rate to zero, not the actual teleportation protocol.

\section{B. NV-center teleportation scheme}

In our second example we consider a nitrogen-vacancy (NV) center in diamond - a point defect in the diamond lattice which consists of a substitutional $\mathrm{N}$ atom and a neighboring vacancy site (see Fig. 2). NV centers are promising candidates for implementing quantum technologies since they exhibit atom like properties in a solid-state environment [40,41]. It is their long-lived spin quantum states which can be addressed via optical transitions that make them particularly attractive as solid-state spin qubits. Numerous experiments involving NV centers have successfully been carried out in recent years [42-44], among them a quantum teleportation between distant NV centers [45]. Here, we investigate a teleportation scheme within a single neutral $\mathrm{NV}^{0}$ center. While experiments usually concentrate on the negatively charged $\mathrm{NV}^{-}$, also the neutral $\mathrm{NV}^{0}$ has recently attracted attention [46-48]. The teleportation scheme is inspired by the work in Ref. [39], where methods for reliable teleportation involving interactions among the involved quantum particles are investigated.
Our proposed $\mathrm{NV}^{0}$ teleportation scheme involves five electrons in eight spin-orbitals, which corresponds to three holes residing in the dangling-bond orbitals around a vacancy site in diamond (see Fig. 2 for a schematic visualization). More specifically, the three $\mathrm{C}$ atoms nearest to the vacancy site each contribute with a dangling $p$ orbital which hybridize and form three molecular orbitals with angular momentum $m_{l}=-1,0,1$. The $\mathrm{N}$ atom contributes with one dangling $p$ orbital with angular momentum $m_{l}=0$. We start out with an entangled pair of holes in $\mathrm{NV}^{0}$. We assume that this pair of holes is prepared in the following Bell state

$$
\left|\psi_{1}\right\rangle=\frac{1}{\sqrt{2}}\left(\hat{c}_{N \downarrow} \hat{c}_{1 \downarrow}+\hat{c}_{N \uparrow} \hat{c}_{-1 \downarrow}\right)|\mathbf{1}\rangle,
$$

where $\hat{c}_{i}$ are annihilation operators which annihilate an electron, i.e., create a hole, in the respective spin-orbital $i$. For simplicity, the annihilation operators for the $\mathrm{C}$ spin-orbitals do not carry a subscript $C$. $|\mathbf{1}\rangle$ represents the filled state with fully occupied spin-orbitals. $\left|\psi_{1}\right\rangle$ is a particle entangled state. Its maximum overlap with a Fock state is $1 / 2$ which yields a geometric entanglement measure of $E_{G}=1-1 / 2=1 / 2$. Its one-particle reduced density matrix, which in the basis $\{\mathrm{N} \downarrow, \mathrm{N} \uparrow, 1 \downarrow,-1 \downarrow\}$ reads

$$
\rho^{(1 \mathrm{p})}=\frac{1}{2}\left(\begin{array}{llll}
1 & 0 & 0 & 0 \\
0 & 1 & 0 & 0 \\
0 & 0 & 1 & 0 \\
0 & 0 & 0 & 1
\end{array}\right),
$$

gives an entropic particle entanglement of $S\left[\rho^{(1 \mathrm{p})}\right]=1$. If we consider a bipartition between the $\mathrm{C}$ orbitals (Alice) and the $\mathrm{N}$ orbitals (Bob), then we obtain a reduced density matrix (in the basis $\left.\left\{|0\rangle, \hat{c}_{N \downarrow}^{\dagger}|0\rangle, \hat{c}_{N \uparrow}^{\dagger}|0\rangle, \hat{c}_{N \downarrow}^{\dagger} \hat{c}_{N \uparrow}^{\dagger}|0\rangle\right\}\right)$

$$
\rho_{N}=\frac{1}{2}\left(\begin{array}{cccc}
0 & 0 & 0 & 0 \\
0 & 1 & 0 & 0 \\
0 & 0 & 1 & 0 \\
0 & 0 & 0 & 0
\end{array}\right),
$$

which yields an entropic mode entanglement of $S\left[\rho_{N}\right]=1 / 2$. Thus - unlike in our first example- here we already start out with a maximally particle and mode entangled Bell state $\left|\psi_{1}\right\rangle$.

The state to be teleported is initially encoded in the spin state of a third hole residing in the $\mathrm{C}, m_{l}=0$ orbital. The state of this hole reads

$$
\left|\psi_{2}\right\rangle=\left(a \hat{c}_{0 \uparrow}+b \hat{c}_{0 \downarrow}\right)|\mathbf{1}\rangle,
$$

where $a$ and $b$ are unknown coefficients, and $|a|^{2}+|b|^{2}=1$. Clearly, $\left|\psi_{2}\right\rangle$ is neither particle entangled nor mode entangled with respect to the bipartition between the $\mathrm{N}$ and the $\mathrm{C}$ orbitals. By combining $\left|\psi_{1}\right\rangle$ and $\left|\psi_{2}\right\rangle$, we obtain the total state of the $\mathrm{NV}^{0}$ which reads

$$
|\psi\rangle=\frac{1}{\sqrt{2}}\left(\hat{c}_{N \downarrow} \hat{c}_{1 \downarrow}+\hat{c}_{N \uparrow} \hat{c}_{-1 \downarrow}\right)\left(a \hat{c}_{0 \uparrow}+b \hat{c}_{0 \downarrow}\right)|\mathbf{1}\rangle .
$$

The particle and mode entanglement of this state are the same as for $\left|\psi_{1}\right\rangle$ in Eq. (39) since $\left|\psi_{2}\right\rangle$ does not add any 
entanglement. We can rewrite Eq. (43) in the following form:

$$
\begin{aligned}
|\psi\rangle= & \frac{1}{2 \sqrt{2}}\left(\hat{c}_{1 \downarrow} \hat{c}_{0 \uparrow}-\hat{c}_{-1 \downarrow} \hat{c}_{0 \downarrow}\right)\left(a \hat{c}_{N \downarrow}-b \hat{c}_{N \uparrow}\right)|\mathbf{1}\rangle \\
& +\frac{1}{2 \sqrt{2}}\left(\hat{c}_{1 \downarrow} \hat{c}_{0 \uparrow}+\hat{c}_{-1 \downarrow} \hat{c}_{0 \downarrow}\right)\left(a \hat{c}_{N \downarrow}+b \hat{c}_{N \uparrow}\right)|\mathbf{1}\rangle \\
& +\frac{1}{2 \sqrt{2}}\left(\hat{c}_{-1 \downarrow} \hat{c}_{0 \uparrow}-\hat{c}_{1 \downarrow} \hat{c}_{0 \downarrow}\right)\left(a \hat{c}_{N \uparrow}-b \hat{c}_{N \downarrow}\right)|\mathbf{1}\rangle \\
& +\frac{1}{2 \sqrt{2}}\left(\hat{c}_{-1 \downarrow} \hat{c}_{0 \uparrow}+\hat{c}_{1 \downarrow} \hat{c}_{0 \downarrow}\right)\left(a \hat{c}_{N \uparrow}+b \hat{c}_{N \downarrow}\right)|\mathbf{1}\rangle,
\end{aligned}
$$

where we have factorized every contribution to $|\psi\rangle$ into two parts (brackets). The second brackets clearly resemble, apart from phase factors (signs), the original state to be teleported of Eq. (42). However, in order to perform the teleportation, we need to be able to distinguish between the four lines of Eq. (44) through a measurement. For this purpose we consider a Coulomb interaction among the holes residing in the $\mathrm{C}$ spin-orbitals. Let us first recall that the Coulomb interaction conserves the total spin and angular momentum. Thus, terms with same spins, such as $\hat{c}_{-1 \downarrow} \hat{c}_{0 \downarrow}$, remain unchanged. Terms with opposite spins can undergo the following spin-flip transitions

$$
\begin{gathered}
\hat{c}_{1 \downarrow} \hat{c}_{0 \uparrow} \longrightarrow \hat{c}_{1 \uparrow} \hat{c}_{0 \downarrow} \\
\hat{c}_{-1 \downarrow} \hat{c}_{0 \uparrow} \longrightarrow \hat{c}_{-1 \uparrow} \hat{c}_{0 \downarrow},
\end{gathered}
$$

where all spins of the involved spin-orbitals are flipped. By applying these spin-flip Coulomb interactions to $|\psi\rangle$, we obtain

$$
\begin{aligned}
\left|\psi^{\prime}\right\rangle= & -\frac{1}{2 \sqrt{2}} \hat{c}_{0 \downarrow}\left(\hat{c}_{1 \uparrow}-\hat{c}_{-1 \downarrow}\right)\left(a \hat{c}_{N \downarrow}-b \hat{c}_{N \uparrow}\right)|\mathbf{1}\rangle \\
& -\frac{1}{2 \sqrt{2}} \hat{c}_{0 \downarrow}\left(\hat{c}_{1 \uparrow}+\hat{c}_{-1 \downarrow}\right)\left(a \hat{c}_{N \downarrow}+b \hat{c}_{N \uparrow}\right)|\mathbf{1}\rangle \\
& -\frac{1}{2 \sqrt{2}} \hat{c}_{0 \downarrow}\left(\hat{c}_{-1 \uparrow}-\hat{c}_{1 \downarrow}\right)\left(a \hat{c}_{N \uparrow}-b \hat{c}_{N \downarrow}\right)|\mathbf{1}\rangle \\
& -\frac{1}{2 \sqrt{2}} \hat{c}_{0 \downarrow}\left(\hat{c}_{-1 \uparrow}+\hat{c}_{1 \downarrow}\right)\left(a \hat{c}_{N \uparrow}+b \hat{c}_{N \downarrow}\right)|\mathbf{1}\rangle .
\end{aligned}
$$

We can now quantify the particle and mode entanglement of $\left|\psi^{\prime}\right\rangle$. The reduced density matrix

$$
\rho_{N}=\frac{1}{2}\left(\begin{array}{llll}
0 & 0 & 0 & 0 \\
0 & 1 & 0 & 0 \\
0 & 0 & 1 & 0 \\
0 & 0 & 0 & 0
\end{array}\right)
$$

gives an entropic mode entanglement of $S\left[\rho_{N}\right]=1 / 2$ for $\left|\psi^{\prime}\right\rangle$, which is the same value as for $|\psi\rangle$. The reason is that the spinflip transitions under consideration in Eq. (45) are all local operations on the $\mathrm{C}$ orbitals while the $\mathrm{N}$ orbital is not affected. The particle-number superselection rule does not change the mode entanglement of $\left|\psi^{\prime}\right\rangle$ since in any possible outcome of a measurement [all four lines in Eq. (46)] there are one hole in the $\mathrm{N}$ orbitals (Bob) and two holes in the $\mathrm{C}$ orbitals (Alice).

The spin-flip transitions in Eq. (45) are particle interactions which may change the particle entanglement in $\left|\psi^{\prime}\right\rangle$. To explicitly calculate the particle entanglement of $\left|\psi^{\prime}\right\rangle$, we construct its one-particle reduced density matrix in the basis $\{0 \downarrow, 1 \downarrow, 1 \uparrow,-1 \downarrow,-1 \uparrow, \mathrm{N} \downarrow, \mathrm{N} \uparrow\}$

$$
\rho^{(1 \mathrm{p})}=\frac{1}{2}\left(\begin{array}{ccccccc}
2 & 0 & 0 & 0 & 0 & 0 & 0 \\
0 & |b|^{2} & a^{*} b & 0 & 0 & 0 & 0 \\
0 & a b^{*} & |a|^{2} & 0 & 0 & 0 & 0 \\
0 & 0 & 0 & |b|^{2} & a^{*} b & 0 & 0 \\
0 & 0 & 0 & a b^{*} & |a|^{2} & 0 & 0 \\
0 & 0 & 0 & 0 & 0 & 1 & 0 \\
0 & 0 & 0 & 0 & 0 & 0 & 1
\end{array}\right)
$$

and find an entropic particle entanglement of $S\left[\rho^{(1 \mathrm{p})}\right]=$ $1 / 2\left[1+2\left(|a|^{2}+|b|^{2}\right)-\left(|a|^{2}+|b|^{2}\right)^{2}\right]=1$. Surprisingly, this is the same value as for the state $|\psi\rangle$. The geometric particle entanglement stays also the same, i.e., $E_{G}=1 / 2$. By taking a second look at the effect of the spin-flip interactions in Eq. (45) we find that for $|\psi\rangle$ they simply act as a unitary spin transformation given by

$$
\begin{aligned}
\hat{c}_{1 \downarrow} & \rightarrow b \hat{c}_{1 \downarrow}+a \hat{c}_{1 \uparrow} \quad \hat{c}_{1 \uparrow} \rightarrow-a \hat{c}_{1 \downarrow}+b \hat{c}_{1 \uparrow} \\
\hat{c}_{-1 \downarrow} & \rightarrow b \hat{c}_{-1 \downarrow}+a \hat{c}_{-1 \uparrow} \quad \hat{c}_{-1 \uparrow} \rightarrow-a \hat{c}_{-1 \downarrow}+b \hat{c}_{-1 \uparrow} \\
\hat{c}_{0 \downarrow} & \rightarrow b \hat{c}_{0 \downarrow}-a \hat{c}_{0 \uparrow} \quad \hat{c}_{0 \uparrow} \rightarrow a \hat{c}_{0 \downarrow}+b \hat{c}_{0 \uparrow}
\end{aligned}
$$

which brings $|\psi\rangle$ to $\left|\psi^{\prime}\right\rangle$. This explains why the particle entanglement is the same in $|\psi\rangle$ and $\left|\psi^{\prime}\right\rangle$.

The spin-flip transition localize one hole in the $\mathrm{C} 0 \downarrow$ orbital. Alice can therefore distinguish between the four different lines in Eq. (46) by measuring the occupation of the $m_{l}= \pm 1$ spin-orbitals. According to the outcome of this measurement she can tell Bob to apply the appropriate spin rotation and phase shift to the $\mathrm{N}$ orbital to obtain the original state to be teleported.

The initial Bell state $\left(\left|\psi_{2}\right\rangle\right)$ is both maximally particle entangled and N-SSR mode entangled, which allows this scheme to reach a $100 \%$ success rate. The spin-flip transitions do not change the particle entanglement of $\left|\psi^{\prime}\right\rangle$ nor the mode entanglement between Alice and Bob, but they allow Alice to perform a less involved orbital occupation measurement instead of a Bell state measurement that would require a particle entangled reference state. Such an orbital-selective occupation measurement should be feasible with the current experimental accuracy in manipulating NV centers. However, the preparation of the highly entangled resource state in Eq. (39) still poses a formidable challenge for the experimental realization of this teleportation protocol.

\section{Teleportation in a quantum dot array}

Semiconductor quantum dots are another promising realization of solid-state qubits for quantum computation [6,49]. In a quantum dot, the qubit is usually represented by the spin degree of freedom of an excess electron since the decoherence time of the spin is much longer than that of the charge [50]. Several protocols for quantum information processing or coherent spin transport with quantum dots have already been proposed and implemented [50-53]. Also a few teleportation schemes based on quantum dot arrays have been suggested in Refs. [54-56]. Inspired by these references, we here present 
(a)

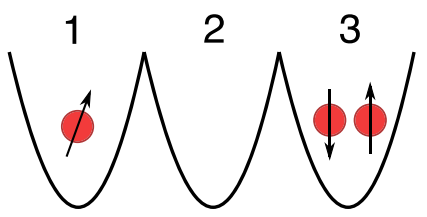

(b)

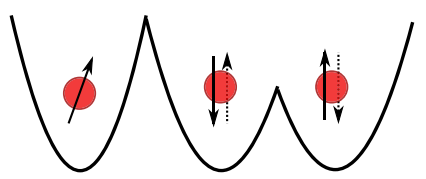

(c)

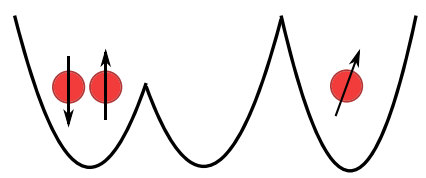

FIG. 3. Teleportation scheme with three quantum dots: (a) The state to be teleported, Eq. (50), is initially encoded in the spin state of an electron residing in dot 1 . Two additional electrons in a singlet state are located in dot 3. (b) A particle-entangled support state, Eq. (52), is created through tunneling between dot 2 and 3. (c) One possible outcome of a successful teleportation [see first line of Eq. (59)] obtained through a second tunneling process between dot 1 and 2 . The state to be teleported is now located in dot 3 .

as a third example a teleportation scheme involving a linear array of three quantum dots.

In our scheme, the state to be teleported is initially encoded in the spin state of an electron in the first dot of the array [see Fig. 3(a)]. This state reads

$$
\left|\psi_{1}\right\rangle=\left(\alpha \hat{c}_{1 \uparrow}^{\dagger}+\beta \hat{c}_{1 \downarrow}^{\dagger}\right)|0\rangle,
$$

where $\alpha$ and $\beta$ are unknown coefficients and $|\alpha|^{2}+|\beta|^{2}=1$. $\left|\psi_{1}\right\rangle$ is neither particle nor mode entangled, since we assign dot 1 and 2 to Alice and dot 3 to Bob. Two additional electrons are residing in the third dot, i.e.,

$$
\left|\psi_{2}\right\rangle=\hat{c}_{3 \uparrow}^{\dagger} \hat{c}_{3 \downarrow}^{\dagger}|0\rangle .
$$

These two electrons are used to produce an entangled resource state via a conditional tunneling between dot 2 and 3 . This tunneling process can be controlled by lowering and raising the tunnel barrier between the dots through a gate voltage [49]. Through the tunneling, which is assisted by the local Coulomb interaction within the dots or by a total occupation measurement of dot 2 or dot 3 , we can obtain the following entangled resource state [see Fig. 3(b)]:

$$
\left|\psi_{2}^{\prime}\right\rangle=\frac{1}{\sqrt{2}}\left(\hat{c}_{2 \uparrow}^{\dagger} \hat{c}_{3 \downarrow}^{\dagger}-\hat{c}_{2 \downarrow}^{\dagger} \hat{c}_{3 \uparrow}^{\dagger}\right)|0\rangle .
$$

Similar to Eq. (39) in the second example, $\left|\psi_{2}^{\prime}\right\rangle$ represents a maximally particle-entangled state of two electrons with a geometric entanglement measure of $E_{G}=1 / 2$ and a particle entanglement entropy of $S=1$. With a bipartition between dot 2 and 3, the mode entanglement as well as the N-SSR restricted mode entanglement of $\left|\psi_{2}^{\prime}\right\rangle$ can easily be evaluated as $S=1 / 2$.
By combining $\left|\psi_{1}\right\rangle$ and $\left|\psi_{2}^{\prime}\right\rangle$, we can write the total state of the quantum dot array as

$$
\begin{aligned}
|\psi\rangle= & \frac{1}{\sqrt{2}}\left(\alpha \hat{c}_{1 \uparrow}^{\dagger}+\beta \hat{c}_{1 \downarrow}^{\dagger}\right)\left(\hat{c}_{2 \uparrow}^{\dagger} \hat{c}_{3 \downarrow}^{\dagger}-\hat{c}_{2 \downarrow}^{\dagger} \hat{c}_{3 \uparrow}^{\dagger}\right)|0\rangle \\
= & \frac{1}{\sqrt{2}}\left(-\alpha \hat{c}_{1 \uparrow}^{\dagger} \hat{c}_{2 \downarrow}^{\dagger} \hat{c}_{3 \uparrow}^{\dagger}+\beta \hat{c}_{1 \downarrow}^{\dagger} \hat{c}_{2 \uparrow}^{\dagger} \hat{c}_{3 \downarrow}^{\dagger}\right. \\
& \left.+\alpha \hat{c}_{1 \uparrow}^{\dagger} \hat{c}_{2 \uparrow}^{\dagger} \hat{c}_{3 \downarrow}^{\dagger}-\beta \hat{c}_{1 \downarrow}^{\dagger} \hat{c}_{2 \downarrow}^{\dagger} \hat{c}_{3 \uparrow}^{\dagger}\right)|0\rangle .
\end{aligned}
$$

The entanglement of $|\psi\rangle$ is the same as for $\left|\psi_{2}^{\prime}\right\rangle$ since $\left|\psi_{1}\right\rangle$ does not add any entanglement. In the next step, we allow tunneling between dot 1 and 2 . In this way, we couple the state to be teleported residing in dot 1 to the entangled resource state in dots 2 and 3. First, we note that the two expressions in the last line of Eq. (53) do not allow for any tunneling between dot 1 and 2 since the electrons have the same spin. The expressions in the second line of Eq. (53) allow instead for tunneling. If both electrons reside in either dot 1 or 2 in the end, then we can achieve a $100 \%$ success rate. To this end we choose a particular conditional tunneling process given by the following conditional hopping operator which is diagonal in the bonding/antibonding basis defined in Eq. (17),

$$
\begin{aligned}
\hat{H}= & \frac{U}{4} \hat{c}_{a \uparrow}^{\dagger} \hat{c}_{a \downarrow}^{\dagger} \hat{c}_{a \downarrow} \hat{c}_{a \uparrow}+\frac{3 U}{4} \hat{c}_{a \downarrow}^{\dagger} \hat{c}_{b \uparrow}^{\dagger} \hat{c}_{b \uparrow} \hat{c}_{a \downarrow} \\
& +\frac{5 U}{4} \hat{c}_{b \uparrow}^{\dagger} \hat{c}_{b \downarrow}^{\dagger} \hat{c}_{b \downarrow} \hat{c}_{b \uparrow}+\frac{7 U}{4} \hat{c}_{a \uparrow}^{\dagger} \hat{c}_{b \downarrow}^{\dagger} \hat{c}_{b \downarrow} \hat{c}_{a \uparrow} .
\end{aligned}
$$

In order to apply the evolution operator $\hat{W}^{(2)}(t=\pi / U)$ to $|\psi\rangle$ according to Eq. (15) we first need to write the creation operators in $|\psi\rangle$ to the $a b$-basis,

$$
\begin{aligned}
|\psi\rangle= & \left(\frac{-1}{2 \sqrt{2}} \hat{c}_{a \uparrow}^{\dagger} \hat{c}_{a \downarrow}^{\dagger}+\frac{1}{2 \sqrt{2}} \hat{c}_{b \uparrow}^{\dagger} \hat{c}_{b \downarrow}^{\dagger}\right)\left(\alpha \hat{c}_{3 \uparrow}^{\dagger}+\beta \hat{c}_{3 \downarrow}^{\dagger}\right) \\
& +\left(\frac{1}{2 \sqrt{2}} \hat{c}_{a \downarrow}^{\dagger} \hat{c}_{b \uparrow}^{\dagger}+\frac{1}{2 \sqrt{2}} \hat{c}_{a \uparrow}^{\dagger} \hat{c}_{b \downarrow}^{\dagger}\right)\left(\alpha \hat{c}_{3 \uparrow}^{\dagger}-\beta \hat{c}_{3 \downarrow}^{\dagger}\right) \\
& -\frac{\alpha}{\sqrt{2}} \hat{c}_{a \uparrow}^{\dagger} \hat{c}_{b \uparrow}^{\dagger} \hat{c}_{3 \downarrow}^{\dagger}+\frac{\beta}{\sqrt{2}} \hat{c}_{a \downarrow}^{\dagger} \hat{c}_{b \downarrow}^{\dagger} \hat{c}_{3 \uparrow}^{\dagger}|0\rangle .
\end{aligned}
$$

We can now directly apply the operator $W^{(2)}(t=\pi / U)$, giving

$$
\begin{aligned}
\left|\psi^{\prime}\right\rangle= & \left(\frac{-e^{\frac{i \pi}{4}}}{2 \sqrt{2}} \hat{c}_{a \uparrow}^{\dagger} \hat{c}_{a \downarrow}^{\dagger}+\frac{e^{\frac{-3 i \pi}{4}}}{2 \sqrt{2}} \hat{c}_{b \uparrow}^{\dagger} \hat{c}_{b \downarrow}^{\dagger}\right)\left(\alpha \hat{c}_{3 \uparrow}^{\dagger}+\beta \hat{c}_{3 \downarrow}^{\dagger}\right) \\
& +\left(\frac{e^{\frac{3 i \pi}{4}}}{2 \sqrt{2}} \hat{c}_{a \downarrow}^{\dagger} \hat{c}_{b \uparrow}^{\dagger}+\frac{e^{-\frac{i \pi}{4}}}{2 \sqrt{2}} \hat{c}_{a \uparrow}^{\dagger} \hat{c}_{b \downarrow}^{\dagger}\right)\left(\alpha \hat{c}_{3 \uparrow}^{\dagger}-\beta \hat{c}_{3 \downarrow}^{\dagger}\right) \\
& -\frac{\alpha}{\sqrt{2}} \hat{c}_{a \uparrow}^{\dagger} \hat{c}_{b \uparrow}^{\dagger} \hat{c}_{3 \downarrow}^{\dagger}+\frac{\beta}{\sqrt{2}} \hat{c}_{a \downarrow}^{\dagger} \hat{c}_{b \downarrow}^{\dagger} \hat{c}_{3 \uparrow}^{\dagger}|0\rangle .
\end{aligned}
$$

Transforming the creation operators in $\left|\psi^{\prime}\right\rangle$ back to the quantum dot basis yields

$$
\begin{aligned}
\left|\psi^{\prime}\right\rangle= & \frac{-1}{2}\left[\hat{c}_{1 \uparrow}^{\dagger} \hat{c}_{1 \downarrow}^{\dagger}\left(i \alpha \hat{c}_{3 \uparrow}^{\dagger}+\beta \hat{c}_{3 \downarrow}^{\dagger}\right)+\hat{c}_{2 \uparrow}^{\dagger} \hat{c}_{2 \downarrow}^{\dagger}\left(\alpha \hat{c}_{3 \uparrow}^{\dagger}+i \beta \hat{c}_{3 \downarrow}^{\dagger}\right)\right]|0\rangle \\
& +\frac{1}{\sqrt{2}}\left(\alpha \hat{c}_{1 \uparrow}^{\dagger} \hat{c}_{2 \uparrow}^{\dagger} \hat{c}_{3 \downarrow}^{\dagger}-\beta \hat{c}_{1 \downarrow}^{\dagger} \hat{c}_{2 \downarrow}^{\dagger} \hat{c}_{3 \uparrow}^{\dagger}\right)|0\rangle .
\end{aligned}
$$

To analyze the particle entanglement after this second tunneling process, we construct the one-particle reduced density 
matrix of $\left|\psi^{\prime}\right\rangle$ in the basis $\{1 \uparrow, 1 \downarrow, 2 \uparrow, 2 \downarrow, 3 \uparrow, 3 \downarrow\}$

$$
\rho^{(1 \mathrm{p})}=\left(\begin{array}{cccccc}
\frac{1+2|\alpha|^{2}}{4} & 0 & 0 & -\frac{i \beta^{*} \alpha^{*}}{\sqrt{2}} & 0 & 0 \\
0 & \frac{1+2|\beta|^{2}}{4} & 0 & 0 & 0 & 0 \\
0 & 0 & \frac{1+2|\alpha|^{2}}{4} & 0 & 0 & 0 \\
\frac{i \beta \alpha}{\sqrt{2}} & 0 & 0 & \frac{1+2|\beta|^{2}}{4} & 0 & 0 \\
0 & 0 & 0 & 0 & \frac{1}{2} & 0 \\
0 & 0 & 0 & 0 & 0 & \frac{1}{2}
\end{array}\right)
$$

and calculate the particle entanglement entropy $S\left[\rho^{(1 \mathrm{p})}\right]=$ $5 / 4$. The geometric entanglement measure $E_{G}\left[\left|\psi^{\prime}\right\rangle\right]=1 / 2$ is obtained from a parameterized search over all Fock states. The particle entanglement entropy is larger than the one for $|\psi\rangle$, i.e., $S=1$, but the geometric entanglement measure is unchanged. The mode entanglement does not change in the tunneling process since the tunneling Hamiltonian only acts on Alice orbitals. Also the particle-number superselection rule does not change the mode entanglement in this teleportation scheme since in any measurement outcome of $\left|\psi^{\prime}\right\rangle$ in Eq. (57) we have two electrons residing with Alice (dot 1 and 2) and one with Bob (dot 3$)$.

The first line of Eq. (57) already resembles our target state for a successful teleportation, with the state to be teleported eventually residing in dot 3 . In order to make use also of the second part of $\left|\psi^{\prime}\right\rangle$, we need to rewrite Eq. (57) in terms of the spin in $x$ direction, i.e., $\hat{c}_{\uparrow(\downarrow)}^{\dagger}=1 / \sqrt{2}\left(\hat{c}_{\uparrow x}^{\dagger}\left({ }_{-}^{+}\right) \hat{c}_{\downarrow x}^{\dagger}\right)$. This yields

$$
\begin{aligned}
\left|\psi^{\prime}\right\rangle= & \frac{1}{2} \hat{c}_{1 \uparrow x}^{\dagger} \hat{c}_{1 \downarrow x}^{\dagger}\left(i \alpha \hat{c}_{3 \uparrow}^{\dagger}+\beta \hat{c}_{3 \downarrow}^{\dagger}\right)|0\rangle \\
& +\frac{1}{2} \hat{c}_{2 \uparrow x}^{\dagger} \hat{c}_{2 \downarrow x}^{\dagger}\left(\alpha \hat{c}_{3 \uparrow}^{\dagger}+i \beta \hat{c}_{3 \downarrow}^{\dagger}\right)|0\rangle \\
& +\frac{1}{2 \sqrt{2}} \hat{c}_{1 \uparrow x}^{\dagger} \hat{c}_{2 \uparrow x}^{\dagger}\left(\alpha \hat{c}_{3 \downarrow}^{\dagger}-\beta \hat{c}_{3 \uparrow}^{\dagger}\right)|0\rangle \\
& +\frac{1}{2 \sqrt{2}} \hat{c}_{1 \downarrow x}^{\dagger} \hat{c}_{2 \uparrow x}^{\dagger}\left(\alpha \hat{c}_{3 \downarrow}^{\dagger}+\beta \hat{c}_{3 \uparrow}^{\dagger}\right)|0\rangle \\
& +\frac{1}{2 \sqrt{2}} \hat{c}_{1 \uparrow x}^{\dagger} \hat{c}_{2 \downarrow x}^{\dagger}\left(\alpha \hat{c}_{3 \downarrow}^{\dagger}+\beta \hat{c}_{3 \uparrow}^{\dagger}\right)|0\rangle \\
& +\frac{1}{2 \sqrt{2}} \hat{c}_{1 \downarrow x}^{\dagger} \hat{c}_{2 \downarrow x}^{\dagger}\left(\alpha \hat{c}_{3 \downarrow}^{\dagger}-\beta \hat{c}_{3 \uparrow}^{\dagger}\right)|0\rangle,
\end{aligned}
$$

where we can now clearly identify in each line the state to be teleported-residing in dot 3 and differing from the original state of Eq. (50) only through a unitary spin rotation. To complete the teleportation, Alice needs to perform a charge and/or magnetization measurement on dot 1 in order to distinguish between the different lines of Eq. (59). According to the outcome of this measurement Bob then needs to apply a magnetic field to the electron spin in dot 3 which yields the original state to be teleported. In Fig. 3(c) we show one possible outcome of a successful teleportation [corresponding to the first line of Eq. (59)] with two electrons residing in quantum dot 1 and the state to be teleported in dot 3 .

Given the fast progress in manipulating spin quantum dots [56,57], an experimental realization of the presented quantum dot teleportation scheme seems feasible in the near future. The most challenging part is the realization of the conditional tunneling operation outlined in Eq. (54). This particular tunneling process could, however, be replaced by a standard tunneling process and a subsequent occupation measurement, at the cost of a lower teleportation success rate.

\section{CONCLUSION AND OUTLOOK}

We have studied the basic mechanisms that generate orbital (mode) and particle entanglement between the electrons in a material. Mode entanglement is defined with respect to a bipartition of orbitals and is closely related to the entanglement of distinguishable particles. It is formed both in nonlocal electron hopping processes and due to the Coulomb interaction between the electrons in a material. Particle entanglement refers to the quantum correlations in a fermionic state which cannot be described by a Fock state (single Slater determinant). It is formed through electron-electron (Coulomb) interaction, both within a material and during a measurement process. An ideal photoemission measurement (photon in, electron out) does not generate particle entanglement within the system, but a spectroscopic measurement (photon in, photon out), such as resonant inelastic x-ray scattering, may increase the particle entanglement in the system.

The N-SSR restricted mode entanglement measure contains a projective measurement of the number of electrons in a local orbital partition. We have shown (in Appendix C) that if an N-electon state has N-SSR restricted mode entanglement, then it will also be particle entangled after the projective particle number measurement. The N-SSR restricted mode entanglement is, however, not directly connected to a difference in particle entanglement before and after the measurement, since the measurement can not only create particle entanglement but also destroy it.

We have proposed three solid-state quantum teleportation schemes and investigated the formation and the role of particle- and mode entanglement therein. (i) Our first protocol teleports an electronic state within a hydrogen molecule on graphene. It only requires that the system starts in a mode entangled state but not a particle-entangled state. The protocol creates particle entanglement through a magnetization measurement, but this particle entanglement is shared between the system and the detector and vanish when the detector is projected out. The lack of a particle entangled state within the system itself comes at the cost of a strongly reduced success rate $(50 \%)$. The spin-orbitals can be bipartitioned in two different ways without affecting the teleportation protocol and its success rate. The two choices does, however, affect the calculated mode entanglement and N-SSR restricted mode entanglement. When the final state is local one may perform a local particle number measurement either at the start of the protocol or after the verification without affecting the total success rate of the teleportation. The N-SSR restricted mode entanglement incorporates the additional information provided by this occupation measurement, which allows it to better reflect the optimal success rate of a quantum teleportation protocol with a local final state, compared to the unrestricted mode entanglement. On the other hand, the $\mathrm{N}$ SSR restricted mode entanglement (severely) underestimates the success rate of the teleportation protocol if the final tele- 
ported state is mode entangled, i.e., if the verification of a successful teleportation requires a nonlocal measurement.

(ii) The second teleportation scheme uses a neutral nitrogen-vacancy center $\mathrm{NV}^{0}$ in diamond. The initial state is a highly mode and particle entangled state with one hole in the nitrogen orbital (belonging to Bob) and two holes in the carbon orbitals (belonging to Alice) [Eq. (43)]. The local integer particle number is conserved throughout the teleportation scheme, and both the initial mode and particle entanglement is preserved in the teleportation process until the final measurements by Alice. This protocol utilizes that the Coulomb interaction between two electrons can cause them to exchange their spins, which yields a $100 \%$ success rate in the ideal case without the need for a Bell-type measurement. (iii) Our third example describes a quantum teleportation in a quantum dot array. A particle and mode entangled state [Eq. (52)] is initially created through a conditional tunneling process between two quantum dots. Also this example yields a $100 \%$ success rate in the ideal case without the need for a Bell-type measurement through the use of a conditional hopping process.

Thus, in the investigated teleportation schemes both mode and particle entanglement are present and play an important role. Nevertheless, the state of the system (not including the detector) does not have to be particle entangled for a quantum teleportation to be successful $50 \%$ of the times. For a $100 \%$ teleportation success rate instead one needs to use a Bell state which is maximally particle and mode entangled. The initial $\mathrm{N}-\mathrm{SSR}$ restricted mode entanglement reflects the optimal success rate of the set of quantum teleportation protocols that start with a local particle number measurement. However, this set of protocols does not in general contain the optimal teleportation protocol if the final teleported state is mode entangled. These results support our view that the different forms of entanglement between electrons are complementary, and that there is no single definition of entanglement that captures all aspects of a quantum system or a quantum protocol.

\section{ACKNOWLEDGMENTS}

We thank Sumanta Bhandary and Jakob Steinbauer for fruitful discussions. A.G. acknowledges support through Schrödinger fellowship J-4267 of the Austrian Science Fund (FWF) and through a "Sub auspiciis Exzellenzstipendium" of the Austrian Federal Ministry of Education, Science and Research. P.T. acknowledges support from Carl Trygger's Foundation, Grant No. CTS 18:389.

\section{APPENDIX A: PARTICLE AND MODE ENTANGLEMENT}

\section{Second quantization formalism}

The second quantization formalism offers a natural way to address states of identical particles. It is based on the notion of creation $\left(\hat{c}_{i}^{\dagger}\right)$ and annihilation $\left(\hat{c}_{i}\right)$ operators, which create and destroy an electron in the spin-orbital $i$, respectively. In the following we will only consider orthonormalized orbitals, to avoid the additional algebra associated with overlap matrices.

All pure many-body states can be formed by applying the creation operators to the vacuum state $(|0\rangle)$, which is annihilated by any annihilation operator, $\hat{c}_{i}|0\rangle \equiv 0$. A pure
$N$-electron state [58] can hence be written

$$
|\psi\rangle=\sum_{\mathbf{i} \in \mathcal{S}_{N}} A_{\mathbf{i}} \hat{S}_{\mathbf{i}}^{\dagger}|0\rangle,
$$

where the Slater determinant index $\mathbf{i} \in \mathcal{S}_{N}$ is an ordered sequence of $N$ orbital indices, i.e., $\mathbf{i}_{1}<\mathbf{i}_{2}<\cdots<\mathbf{i}_{N}$, and $\hat{S}_{\mathbf{i}}^{\dagger} \equiv$ $\hat{c}_{\mathbf{i}_{1}}^{\dagger} \hat{c}_{\mathbf{i}_{2}}^{\dagger} \cdots \hat{c}_{\mathbf{i}_{N}}^{\dagger}$. A state that can be written as $\hat{S}_{\mathbf{i}}^{\dagger}|0\rangle$ in a given orbital basis is known as a Slater determinant. In the following we will call a state that is a Slater determinant in some orbital basis, but not necessarily the given one, a Fock state. The reason for this distinction will become clear when we define particle and mode entanglement.

The choice of orbital basis does not hold any physical significance in itself. A change of the orbital basis $\hat{c}_{i}^{\dagger} \rightarrow \hat{c}_{i}^{\prime \dagger}$, where

$$
\hat{c}_{j}^{\prime \dagger} \equiv \sum_{i} \hat{c}_{i}^{\dagger} V_{i j}^{\dagger},
$$

and $V$ is a unitary transformation, can be performed by substituting the identity

$$
\hat{c}_{i}^{\dagger}=\sum_{j} \hat{c}_{j}^{\prime \dagger} V_{j i}
$$

into Eq. (A1). The fermionic commutation relation $\hat{c}_{i}^{\dagger} \hat{c}_{j}^{\dagger}=$ $-\hat{c}_{j}^{\dagger} \hat{c}_{i}^{\dagger}$ can then be used to sort the creation operators according to the selected orbital order. For example, the state

$$
\left|\psi^{\prime}\right\rangle=\frac{1}{2}\left(\hat{c}_{1 \uparrow}^{\dagger}+\hat{c}_{1 \downarrow}^{\dagger}\right)\left(\hat{c}_{2 \uparrow}^{\dagger}+\hat{c}_{2 \downarrow}^{\dagger}\right)|0\rangle,
$$

represents two electrons located in the spin-orbitals $1 \uparrow, 1 \downarrow$, $2 \uparrow$, and $2 \downarrow$. In the orbital basis

$$
\begin{array}{ll}
\hat{c}_{1 \uparrow x}^{\dagger}=\frac{1}{\sqrt{2}}\left(\hat{c}_{1 \uparrow}^{\dagger}+\hat{c}_{1 \downarrow}^{\dagger}\right) & \hat{c}_{2 \uparrow x}^{\dagger}=\frac{1}{\sqrt{2}}\left(\hat{c}_{2 \uparrow}^{\dagger}+\hat{c}_{2 \downarrow}^{\dagger}\right) \\
\hat{c}_{1 \downarrow x}^{\dagger}=\frac{1}{\sqrt{2}}\left(\hat{c}_{1 \uparrow}^{\dagger}-\hat{c}_{1 \downarrow}^{\dagger}\right) & \hat{c}_{2 \downarrow x}^{\dagger}=\frac{1}{\sqrt{2}}\left(\hat{c}_{2 \uparrow}^{\dagger}-\hat{c}_{2 \downarrow}^{\dagger}\right),
\end{array}
$$

it takes the simple product form

$$
\left|\psi^{\prime}\right\rangle=\hat{c}_{1 \uparrow x}^{\dagger} \hat{c}_{2 \uparrow x}^{\dagger}|0\rangle .
$$

The unitary transformation in Eq. (A5) corresponds to a $\pi / 2$ spin rotation around the $y$ axis.

\section{Particle entanglement}

The product form of a Fock state, i.e., that it can be written as a single Slater determinant $\hat{S}_{\mathbf{i}}^{\dagger}|0\rangle=\hat{c}_{i_{1}}^{\dagger} \hat{c}_{i_{2}}^{\dagger} \cdots \hat{c}_{i_{N}}^{\dagger}|0\rangle$ in some orbital basis, gives it properties closely related to those of a product state of distinguishable particles [20,24]. For example, an unknown Fock state can be fully characterized by single-particle measurements of the orbital occupations. A Fock state can therefore in principle be described by a hidden-variable theory, where the hidden variables specify which orbitals are fully occupied. This has lead to the concept of particle entanglement, which identifies the Fock states as being nonentangled [19,20,22-24,24,59]. It should, however, be noted that it is only when the occupied orbitals of a Fock state are local that the hidden variables description become local as well. The "nonlocal" Fock states can hence still potentially be used as a resource in a quantum computational algorithm. 


\section{Mode entanglement}

Although the choice of orbital basis does not hold any physical significance, the way the state can be written in terms of creation operators still affects orbital-dependent quantities. For example, given a set of orbitals that belongs to Alice $(A)$, and a set that belongs to Bob $(B)$, one may ask whether the electrons within one orbital partition can be described independently of the electrons in the other partition. This is the defining idea behind mode entanglement [8], which follows closely the concept of entanglement between distinguishable particles but applied to the orbital occupation. A state $|\psi\rangle$ is mode entangled with respect to the orbital partitions $A$ and $B$ unless it can be written as the product

$$
|\psi\rangle=\left(\sum_{\mathbf{i} \in \mathcal{S}^{A}} A_{\mathbf{i}} \hat{S}_{\mathbf{i}}^{\dagger}\right)\left(\sum_{\mathbf{j} \in \mathcal{S}^{B}} B_{\mathbf{j}} \hat{S}_{\mathbf{j}}^{\dagger}\right)|0\rangle,
$$

where $\mathbf{i} \in \mathcal{S}^{A}$ denotes all possible combinations of occupied orbitals in $A$. Even a single electron state can thus be mode entangled if the corresponding occupied orbital is shared between $A$ and $B$. The simplest possible mode entangled state is hence

$$
|\psi\rangle=\frac{1}{\sqrt{2}}\left(\hat{c}_{1}^{\dagger}+\hat{c}_{2}^{\dagger}\right)|0\rangle,
$$

where orbital 1 and 2 belong to partition $A$ and $B$, respectively. A less trivial example is given by $\left|\psi^{\prime}\right\rangle$ in Eq. (A4) if the spin up orbitals belong to $A$ and the spin down orbitals to $B$. A third example of a mode entangled state is

$$
\left|\psi^{\prime \prime}\right\rangle=\frac{1}{\sqrt{2}}\left(\hat{c}_{1 \uparrow}^{\dagger} \hat{c}_{2 \downarrow}^{\dagger}+\hat{c}_{1 \downarrow}^{\dagger} \hat{c}_{2 \uparrow}^{\dagger}\right)|0\rangle .
$$

This state is special since it is mode entangled with respect to any bipartition of the spin-orbitals $1 \uparrow, 1 \downarrow, 2 \uparrow$, and $2 \downarrow$. Such state can not be a Fock state, since a fully occupied or empty orbital would form a separable subspace, which implies that the state must be particle entangled [19,20,23,24]. The converse, that a particle entangled state is mode entangled with respect to any bipartition is not true in general since some of the orbitals can still be fully occupied or empty in a particle entangled state.

\section{N-SSR restricted mode entanglement}

A quantum teleportation scheme transfers the unknown state of an electron from one local orbital partition $A$ (belonging to Alice) to another orbital partition $B$ (Bob) without a phase coherent transport of electrons or other information carriers between the two orbital partitions. Only operations that conserve the (local) particle number of the two orbital partitions are hence allowed in the teleportation protocol. The unknown state to be teleported is in general entangled with electrons in a third remote partition, which implies that also its relative phase needs to be transferred. Alice and Bob will need additional electrons to carry out the teleportation, but in order to have well-defined conditions for a successful teleportation these electrons should not initially be entangled with the unknown state. In particular, a successful teleportation should not be affected by any subsequent local measurements performed by Alice. Alice may hence perform a total occupation measurement after the teleportation, without affecting the result, using the local occupation number operator

$$
\hat{N}_{A}=\sum_{i \in A} \hat{c}_{i}^{\dagger} \hat{c}_{i}
$$

where $i \in A$ runs over all the spin-orbitals in $A$. Since all the allowed operators in the teleportation protocol preserve the number of electrons in $A$, they commute with $\hat{N}_{A}$. This implies that Alice may perform a total occupation measurement of her orbital partition at the very start of the teleportation protocol and still get the same result on average [14]. The $\mathrm{N}-\mathrm{SSR}$ restricted mode entanglement corresponds to the mode entanglement of the system but after a projective total occupation measurement of the local orbital partition $A$ or $B$. The measurement of the occupation number projects an N-electron state $\left|\psi_{N}\right\rangle$ into $\hat{P}_{A}^{(n)}\left|\psi_{N}\right\rangle$ with probability $\left\langle\psi_{N}\left|\hat{P}_{A}^{(n)}\right| \psi_{N}\right\rangle$, where the projection operator $\hat{P}_{A}^{(n)}$ is given by

$$
\hat{P}_{A}^{(n)}=\sum_{\substack{\mathbf{i} \in \mathcal{S}^{B} \\ \mathbf{j} \in \mathcal{S}_{n}^{A}}} \hat{S}_{\mathbf{i}}^{\dagger} \hat{P}_{\mathbf{j i}}
$$

where the operator

$$
\hat{P}_{\mathbf{j i}}=\hat{S}_{\mathbf{j}}^{\dagger}|0\rangle\langle 0| \hat{S}_{\mathbf{j}} \hat{S}_{\mathbf{i}}
$$

has been introduced for later convenience. A pure $\mathrm{N}$-electron state is therefore N-SSR mode entangled unless it can be written in the form

$$
\left|\psi_{N}\right\rangle=\sum_{n=0}^{N}\left(\sum_{\mathbf{i} \in \mathcal{S}_{n}^{A}} A_{\mathbf{i}} \hat{S}_{\mathbf{i}}^{\dagger}\right)\left(\sum_{\mathbf{j} \in \mathcal{S}_{N-n}^{B}} B_{\mathbf{j}} \hat{S}_{\mathbf{j}}^{\dagger}\right)|0\rangle .
$$

As shown in Appendix C, if an N-electron state has N-SSR restricted mode entanglement, then it will also be particle entangled after the projective occupation number measurement. $\mathrm{N}-\mathrm{SSR}$ restricted mode entanglement is from this perspective a hybrid of mode entanglement and particle entanglement, where the mode entanglement gives the nonlocal correlation while the particle entanglement provides the handle to access the correlation.

\section{APPENDIX B: ENTANGLEMENT MEASURES}

\section{Mode entanglement}

Since the definition of mode entanglement follows closely the definition of entanglement for distinguishable particles, although applied to the orbital occupation, it can be measured in a similar fashion. Given a bipartition of the orbitals into $A$ and $B$, the partial trace of $|\psi\rangle$ over the orbitals in $B$ gives the reduced density matrix

$$
\rho_{A}=\operatorname{Tr}_{B}[|\psi\rangle\langle\psi|] \equiv \sum_{\substack{\mathbf{i} \in \mathcal{S}^{B} \\ \mathbf{j}, \mathbf{k} \in \mathcal{S}^{A}}} \hat{P}_{\mathbf{j i}}|\psi\rangle\langle\psi| \hat{P}_{\mathbf{i k}}^{\dagger},
$$

where the projection operator $\hat{P}_{\mathrm{ji}}$ was introduced in Eq. (A12). The partial trace converts the mode entanglement in $|\psi\rangle$ between $A$ and $B$ to entropy in $\rho_{A}$. The (linear) entropy $S\left[\rho_{A}\right]=$ $\operatorname{Tr}\left[\rho_{A}\left(1-\rho_{A}\right)\right]$ serves therefore as a measure of the mode entanglement. However, if the initial state is mixed, then its 
nonzero entropy is also partially transferred to $\rho_{A}$, which implies that $S\left[\rho_{A}\right]$ only gives an upper bound to the mode entanglement in this case.

\section{N-SSR restricted mode entanglement}

$\mathrm{N}$-SSR restricted mode entanglement is also measured using the entropy of the reduced density matrix, but as an average after the local occupation has been resolved. The $\mathrm{N}$-SSR restricted mode entanglement is hence given by

$$
S^{N}\left[\rho_{A}\right]=\sum_{n} \operatorname{Tr}\left[\rho_{A}^{(n)}\right] S\left[\frac{\rho_{A}^{(n)}}{\operatorname{Tr}\left[\rho_{A}^{(n)}\right]}\right],
$$

where the particle resolved reduced density matrix $\rho_{A}^{(n)}$ of the $\mathrm{N}$-electron state $|\psi\rangle$ is given by

$$
\rho_{A}^{(n)}=\sum_{\substack{\mathbf{i} \in \mathcal{S}_{N-n}^{B} \\ \mathbf{j}, \mathbf{k} \in \mathcal{S}_{n}^{h}}} \hat{P}_{\mathbf{j i}}|\psi\rangle\langle\psi| \hat{P}_{\mathbf{i k}}^{\dagger} .
$$

\section{Particle entanglement}

The particle entanglement of a pure state $|\psi\rangle$ can also be measured using the entropy of a reduced density matrix [17], but in this case of the one-particle reduced density matrix $\rho_{i j}^{(1 \mathrm{p})}=\left\langle\psi\left|\hat{c}_{j}^{\dagger} \hat{c}_{i}\right| \psi\right\rangle$. The entropy

$$
\begin{aligned}
S[|\psi\rangle] & \equiv\langle\psi\rangle \psi S\left[\frac{\rho^{(1 \mathrm{p})}}{\langle\psi\rangle \psi}\right] \\
& =\langle\psi\rangle \psi \operatorname{Tr}\left\{\frac{\rho^{(1 \mathrm{p})}}{\langle\psi\rangle \psi}\left[1-\frac{\rho^{(1 \mathrm{p})}}{\langle\psi\rangle \psi}\right]\right\},
\end{aligned}
$$

is zero for any Fock state, positive for a particle-entangled state, and invariant under any unitary orbital transformation $c_{i}^{\dagger} \rightarrow c_{i}^{\prime \dagger}$. Again, if the initial state is mixed, then $S\left[\rho^{(1 \mathrm{p})}\right]$ will only give an upper bound to the particle entanglement. An alternative particle entanglement measure is based on the geometric distance to the closest Fock state $[34,60,61]$,

$$
E_{G}[|\psi\rangle]=\langle\psi|| \psi\rangle-\max _{\left|\psi^{\prime}\right\rangle}\left|\left\langle\psi^{\prime}|| \psi\right\rangle\right|^{2},
$$

where $\left|\psi^{\prime}\right\rangle$ is restricted to be a Fock state. If the system has only two electrons, then it is straightforward to show that $E_{G}[|\psi\rangle]=\langle\psi\rangle \psi-\rho_{\max }^{(1 \mathrm{p})}$, where $\rho_{\max }^{(1 \mathrm{p})}$ is the largest eigenvalue of the one-particle reduced density matrix $\rho^{(1 \mathrm{p})}[60]$. In the case of three or more electrons the search for the closest Fock state becomes much more involved, as illustrated by the state

$$
\left|\psi^{\prime \prime \prime}\right\rangle=\frac{1}{\sqrt{3}}\left(\hat{c}_{1 \downarrow}^{\dagger} \hat{c}_{2 \uparrow}^{\dagger} \hat{c}_{3 \uparrow}^{\dagger}-\hat{c}_{1 \uparrow}^{\dagger} \hat{c}_{2 \downarrow}^{\dagger} \hat{c}_{3 \uparrow}^{\dagger}+\hat{c}_{1 \uparrow}^{\dagger} \hat{c}_{2 \uparrow}^{\dagger} \hat{c}_{3 \downarrow}^{\dagger}\right)|0\rangle,
$$

which is composed of three Slater determinants. At first glance, the maximum squared overlap of $\left|\psi^{\prime \prime \prime}\right\rangle$ with a Fock state seems to be $1 / 3$. However, a parameterized search over all unitary orbital transformations [60] yields the transformation

$$
\hat{c}_{i \uparrow}^{\prime \dagger}=\frac{1}{\sqrt{3}}\left(\sqrt{2} \hat{c}_{i \uparrow}^{\dagger}+\hat{c}_{i \downarrow}^{\dagger}\right) \quad \hat{c}_{i \downarrow}^{\prime \dagger}=\frac{1}{\sqrt{3}}\left(-\hat{c}_{i \uparrow}^{\dagger}+\sqrt{2} \hat{c}_{i \downarrow}^{\dagger}\right)
$$

that allows us to rewrite the state in Eq. (B6) as

$$
\begin{aligned}
\left|\psi^{\prime \prime \prime}\right\rangle= & \frac{2}{3} \hat{c}_{1 \uparrow}^{\prime \dagger} \hat{c}_{2 \uparrow}^{\prime \dagger} \hat{c}_{3 \uparrow}^{\prime \dagger}|0\rangle+\frac{1}{3} \hat{c}_{1 \uparrow}^{\prime \dagger} \hat{c}_{2 \downarrow}^{\prime \dagger} \hat{c}_{3 \downarrow}^{\prime \dagger}|0\rangle+\frac{1}{3} \hat{c}_{1 \downarrow}^{\prime \dagger} \hat{c}_{2 \uparrow}^{\prime \dagger} \hat{c}_{3 \downarrow}^{\prime \dagger}|0\rangle \\
& -\frac{1}{3} \hat{c}_{1 \downarrow}^{\prime \dagger} \hat{c}_{2 \downarrow}^{\prime \dagger} \hat{c}_{3 \uparrow}^{\prime \dagger}|0\rangle-\frac{\sqrt{2}}{3} \hat{c}_{1 \downarrow}^{\prime \dagger} \hat{c}_{2 \downarrow}^{\prime \dagger} \hat{c}_{3 \downarrow}^{\prime \dagger}|0\rangle .
\end{aligned}
$$

The first Slater determinant, $\hat{c}_{1 \uparrow}^{\prime \dagger} \hat{c}_{2 \uparrow}^{\prime \dagger} \hat{c}_{3 \uparrow}^{\prime \dagger}|0\rangle$, on the right-hand side of Eq. (B8) has a weight of $2 / 3>1 / \sqrt{3}$. The state $\left|\psi^{\prime \prime \prime}\right\rangle$ is hence particle entangled with a geometric entanglement measure of $E_{G}=1-|2 / 3|^{2}=5 / 9$. The entropic entanglement measure for the same state $\left|\psi^{\prime \prime \prime}\right\rangle$ can be calculated from its one-particle reduced density matrix, which in the basis $\left\{1 \uparrow^{\prime}, 1 \downarrow^{\prime}, 2 \uparrow^{\prime}, 2 \downarrow^{\prime}, 3 \uparrow^{\prime}, 3 \downarrow^{\prime}\right\}$ reads

$$
\rho^{(1 \mathrm{p})}=\frac{1}{9}\left(\begin{array}{cccccc}
5 & -\sqrt{2} & 0 & 0 & 0 & 0 \\
-\sqrt{2} & 4 & 0 & 0 & 0 & 0 \\
0 & 0 & 5 & \sqrt{2} & 0 & 0 \\
0 & 0 & \sqrt{2} & 4 & 0 & 0 \\
0 & 0 & 0 & 0 & 5 & \sqrt{2} \\
0 & 0 & 0 & 0 & \sqrt{2} & 4
\end{array}\right) .
$$

By inserting $\rho^{(1 \mathrm{p})}$ into Eq. (B4) we obtain an entanglement entropy of $S=4 / 3$. For comparison, the two-fermion entangled state of Eq. (A9) has an entanglement entropy of $S=1$ and a geometric entanglement measure of $E_{G}=1-1 / 2=1 / 2$. In the result section we will use these entanglement measures to analyze the mode entanglement and the particle entanglement in three different teleportation schemes involving identical particles.

\section{APPENDIX C: N-SSR AND PARTICLE ENTANGLEMENT}

In this Appendix we show that if an N-electron state $|\psi\rangle$ has N-SSR restricted mode entanglement, then the state of the system after the projective orbital occupation measurement $\hat{P}_{A}^{(n)}|\psi\rangle$ [see Eq. (A11)] is particle entangled for some $n$. To prove this it is enough to show that if the state $\hat{P}_{A}^{(n)}|\psi\rangle$ is a Fock state, then its mode entanglement between orbital partition $A$ and another partition $B$ is zero.

Let us start by looking at the one-particle reduce density matrix

$$
\rho_{i j}^{(1 p)}=\left\langle\psi\left|\hat{P}_{A}^{(n) \dagger} \hat{c}_{j}^{\dagger} \hat{c}_{i} \hat{P}_{A}^{(n)}\right| \psi\right\rangle,
$$

in an orbital basis where the orbitals $i$ and $j$ belong to either partition $A$ or $B$. If $i \in A$ and $j \in B$ or $i \in B$ and $j \in A$, then we get

$$
\hat{P}_{A}^{(n) \dagger} \hat{c}_{j}^{\dagger} \hat{c}_{i} \hat{P}_{A}^{(n)}=0,
$$

which implies that all off-diagonal elements in $\rho^{(1 p)}$ between the orbital partition $A$ and $B$ are zero.

If $\hat{P}_{A}^{(n)}|\psi\rangle$ is a Fock state, then there is an orbital transformation that brings $\rho^{(1 p)}$ into a diagonal form with diagonal elements 1 or 0 . Since $\rho^{(1 p)}$ has no off-diagonal elements between $A$ and $B$ this transformation can be performed without mixing the orbitals of the two partitions. In this new orbital basis we can hence write the state $\hat{P}_{A}^{(n)}|\psi\rangle$ as the clearly nonentangled state $\hat{S}_{\mathbf{a}}^{\dagger} \hat{S}_{\mathbf{b}}^{\dagger}|0\rangle$ where $\mathbf{a} \in \mathcal{S}_{n}^{A}$ and $\mathbf{b} \in \mathcal{S}_{N-n}^{B}$. This 
proves that if $\hat{P}_{A}^{(n)}|\psi\rangle$ has no particle entanglement, then its mode entanglement between $A$ and $B$ is also zero.

\section{APPENDIX D: PARTICLE ENTANGLEMENT INEQUALITY}

In this Appendix we prove the particle entanglement inequality in Eq. (25) explicitly for the linear entropy particle entanglement measure $S[|\psi\rangle]$ and the geometric particle entanglement measure $E_{G}[|\psi\rangle]$.

The initial state in Eq. (25) is

$$
\begin{aligned}
\left|\psi^{i}\right\rangle= & \sqrt{\alpha} e^{i \theta} \hat{c}_{e}^{\dagger}\left|\psi_{e}\right\rangle+\sqrt{1-\alpha}\left|\psi_{s}\right\rangle \\
= & {\left[\sqrt{\alpha} e^{i \theta} \hat{c}_{e}^{\dagger}\left(\sum_{\mathbf{i} \in \mathcal{S}_{N-1}} \psi_{\mathbf{i}}^{e} \hat{S}_{\mathbf{i}}^{\dagger}\right)\right.} \\
& \left.+\sqrt{1-\alpha}\left(\sum_{\mathbf{j} \in \mathcal{S}_{N}} \psi_{\mathbf{j}}^{s} \hat{S}_{\mathbf{j}}^{\dagger}\right)\right]|0\rangle,
\end{aligned}
$$

where the states $\hat{c}_{e}^{\dagger}\left|\psi_{e}\right\rangle$ and $\left|\psi_{s}\right\rangle$ are normalized and contain $\mathrm{N}$ electrons. By definition neither $\left|\psi_{e}\right\rangle$ nor $\left|\psi_{s}\right\rangle$ contain an electron in orbital $e$. This allows us, for convenience, to absorb the relative phase $e^{i \theta}$ between the two states into the definition of $\hat{c}_{e}^{\dagger}$. Furthermore, since $E\left[\left|\psi_{e}\right\rangle\right]=E\left[\hat{c}_{e}^{\dagger}\left|\psi_{e}\right\rangle\right]$ we only need to prove the inequality

$$
\begin{aligned}
& E\left[\sqrt{\alpha} \hat{c}_{e}^{\dagger}\left|\psi_{e}\right\rangle+\sqrt{1-\alpha}\left|\psi_{s}\right\rangle\right] \\
& \quad \geqslant \alpha E\left[\hat{c}_{e}^{\dagger}\left|\psi_{e}\right\rangle\right]+(1-\alpha) E\left[\left|\psi_{s}\right\rangle\right] .
\end{aligned}
$$

\section{Linear entropy measure}

The states $\hat{c}_{e}^{\dagger}\left|\psi_{e}\right\rangle$ and $\left|\psi_{s}\right\rangle$ give rise to three different contributions to the one-particle reduced density matrix, $\rho_{i j}=$ $\left\langle\psi^{i}\left|\hat{c}_{j}^{\dagger} \hat{c}_{i}\right| \psi^{i}\right\rangle$, given by

$$
\begin{gathered}
\rho_{i j}=\alpha \rho_{i j}^{e}+(1-\alpha) \rho_{i j}^{s}+\sqrt{\alpha(1-\alpha)} \rho_{i j}^{\text {off }}, \\
\rho_{i j}^{e}=\left\langle\psi^{e}\left|\hat{c}_{e} \hat{c}_{j}^{\dagger} \hat{c}_{i} \hat{c}_{e}^{\dagger}\right| \psi^{e}\right\rangle, \\
\rho_{i j}^{s}=\left\langle\psi^{s}\left|\hat{c}_{j}^{\dagger} \hat{c}_{i}\right| \psi^{s}\right\rangle, \\
\rho_{i j}^{\text {off }}=\left\langle\psi^{e}\left|\hat{c}_{e} \hat{c}_{j}^{\dagger} \hat{c}_{i}\right| \psi^{s}\right\rangle+\left\langle\psi^{s}\left|\hat{c}_{j}^{\dagger} \hat{c}_{i} \hat{c}_{e}^{\dagger}\right| \psi^{e}\right\rangle .
\end{gathered}
$$

Let us now define the creation operator $\hat{c}_{s}^{\dagger}$ through the equation

$$
\sqrt{\beta} \hat{c}_{s}^{\dagger}|0\rangle=(-1)^{N-1} \sum_{\mathbf{i} \in \mathcal{S}_{N-1}} \psi_{\mathbf{i}}^{e *} \hat{S}_{\mathbf{i}}\left|\psi_{s}\right\rangle,
$$

where $\beta$ is a non-negative normalization constant. The orbitals $e$ and $s$ have to be orthogonal when $\beta>0$ since $\hat{c}_{e}\left|\psi_{s}\right\rangle=0$. In case $\beta=0$ any orbital that is orthogonal to $e$ can be selected to represent the orbital $s$. We are therefore always allowed to include both $e$ and $s$ in our orbital basis, which simplifies Eq. (D6) to yield $\rho_{i j}^{\text {off }}=\sqrt{\beta}\left(\delta_{i e} \delta_{j s}+\delta_{i s} \delta_{j e}\right)$. Finally, by writing $\left|\psi_{e}\right\rangle$ and $\left|\psi_{s}\right\rangle$ as superpositions of states that contain $\hat{c}_{s}^{\dagger}$ and states which do not, it is straightforward to show that $\beta \leqslant \rho_{s s}^{s}\left(1-\rho_{s s}^{e}\right)$.
Let us first deal with the case when $\beta=0$, i.e., $\rho^{\text {off }}=0$. The linear entropy of $\rho$ is then given by

$$
\begin{aligned}
S[\rho]= & \operatorname{Tr}\left\{\alpha \rho^{e}+(1-\alpha) \rho^{s}-\left[\alpha \rho^{e}+(1-\alpha) \rho^{s}\right]^{2}\right\} \\
= & \alpha \operatorname{Tr}\left[\rho^{e}\right]+(1-\alpha) \operatorname{Tr}\left[\rho^{s}\right]-\alpha^{2} \operatorname{Tr}\left[\left(\rho^{e}\right)^{2}\right] \\
& -(1-\alpha)^{2} \operatorname{Tr}\left[\left(\rho^{s}\right)^{2}\right]-2 \alpha(1-\alpha) \operatorname{Tr}\left[\rho^{e} \rho^{s}\right] \\
= & \alpha \operatorname{Tr}\left[\rho^{e}-\left(\rho^{e}\right)^{2}\right]+(1-\alpha) \operatorname{Tr}\left[\rho^{s}-\left(\rho^{s}\right)^{2}\right] \\
& +\alpha(1-\alpha)\left(\operatorname{Tr}\left[\left(\rho^{e}\right)^{2}\right]+\operatorname{Tr}\left[\left(\rho^{e}\right)^{2}\right]-2 \operatorname{Tr}\left[\rho^{e} \rho^{s}\right]\right) .
\end{aligned}
$$

The last term in Eq. (D8) is always larger than zero since the product of two hermitian operators $\hat{A}$ and $\hat{B}$ fulfill the CauchySchwarz inequality

$$
\begin{aligned}
\operatorname{Tr}[\hat{A} \hat{B}] & \leqslant \sqrt{\operatorname{Tr}\left[\hat{A}^{2}\right] \operatorname{Tr}\left[\hat{B}^{2}\right]} \\
& \leqslant \sqrt{\operatorname{Tr}\left[\hat{A}^{2}\right] \operatorname{Tr}\left[\hat{B}^{2}\right]+\frac{1}{4}\left(\operatorname{Tr}\left[\hat{A}^{2}\right]-\operatorname{Tr}\left[\hat{B}^{2}\right]\right)^{2}} \\
& =\frac{1}{2}\left(\operatorname{Tr}\left[\hat{A}^{2}\right]+\operatorname{Tr}\left[\hat{B}^{2}\right]\right),
\end{aligned}
$$

which directly yields

$$
\begin{aligned}
S[\rho] & \geqslant \alpha \operatorname{Tr}\left[\rho^{e}-\left(\rho^{e}\right)^{2}\right]+(1-\alpha) \operatorname{Tr}\left[\rho^{s}-\left(\rho^{s}\right)^{2}\right] \\
& =\alpha S\left[\rho^{e}\right]+(1-\alpha) S\left[\rho^{s}\right]
\end{aligned}
$$

when $\beta=0$.

In case $\beta>0$ we get a finite contribution from $\rho^{\text {off }}$ that reduces the linear entropy of $\rho$. To prove the inequality in Eq. (24) it is therefore not enough to only use the CauchySchwarz inequality in Eq. (D9) but we must treat the effect of $\rho^{\text {off }}$ explicitly. To this aim, let us define the projection matrix

$$
P_{i j}=\delta_{e i} \delta_{e j}+\delta_{s i} \delta_{s j}
$$

that project out the orbital subspace spanned by $e$ and $s$. The linear entropy of $\rho$ can then be written as

$S[\rho]=\operatorname{Tr}\left[P \rho P-(P \rho P)^{2}\right]+\operatorname{Tr}\left[(\rho-P \rho P)-(\rho-P \rho P)^{2}\right]$.

The second term in Eq. (D12), $S[\rho-P \rho P]$, does not contain any contribution from $\rho^{\text {off }}$. It can therefore be expanded as in Eq. (D8) and bound by the inequality in Eq. (D9), which yields

$$
S[\rho-P \rho P] \geqslant \alpha S\left[\rho^{e}-P \rho^{e} P\right]+(1-\alpha) S\left[\rho^{s}-P \rho^{s} P\right] .
$$

The first term in Eq. (D12), $S[P \rho P]$, is explicitly given by

$$
\begin{aligned}
S[P \rho P]= & \alpha S\left[P \rho^{e} P\right]+(1-\alpha) S\left[P \rho^{s} P\right] \\
& +\alpha(1-\alpha)\left\{\operatorname{Tr}\left[\left(P \rho^{e} P\right)^{2}\right]+\operatorname{Tr}\left[\left(P \rho^{s} P\right)^{2}\right]\right. \\
& \left.-2 \operatorname{Tr}\left[P \rho^{e} P \rho^{s} P\right]-2 \operatorname{Tr}\left[\rho^{\mathrm{off}} \rho^{\mathrm{off}}\right]\right\} . \\
= & \alpha S\left[P \rho^{e} P\right]+(1-\alpha) S\left[P \rho^{s} P\right] \\
& +\alpha(1-\alpha)\left[1+\left(\rho_{s s}^{e}-\rho_{s s}^{s}\right)^{2}-2 \beta\right] .
\end{aligned}
$$

Substituting $\beta \leqslant \rho_{s s}^{s}\left(1-\rho_{s s}^{e}\right)$ into Eq. (D14) yields

$$
S[P \rho P] \geqslant \alpha S\left[P \rho^{e} P\right]+(1-\alpha) S\left[P \rho^{s} P\right] .
$$

Finally, combining Eq. (D13) and Eq. (D15) gives

$$
S[\rho] \geqslant \alpha S\left[\rho^{e}\right]+(1-\alpha) S\left[\rho^{s}\right],
$$


which together with Eq. (D10) proves Eq. (D2) for the linear entropy measure for all values of $\beta$.

\section{Geometric measure}

Although both the geometric particle entanglement measure $E_{G}[|\psi\rangle]$ and the linear entropy particle entanglement measure $S[|\psi\rangle]$ are zero if and only if $|\psi\rangle$ is a Fock state, they are not equivalent measures. Eq. (D2) requires therefore a separate proof for $E_{G}$.

Let us start by considering an arbitrary Fock state $\left|\psi^{i \prime \prime}\right\rangle$ with $\mathrm{N}$ electrons. Since $\left|\psi^{i \prime \prime}\right\rangle$ is a Fock state there exists an orbital basis in which it can be written as a single Slater determinant $\left|\psi^{i \prime \prime}\right\rangle=\hat{c}_{1}^{\dagger \prime \prime} \hat{c}_{2}^{\dagger \prime \prime} \cdots \hat{c}_{N}^{\dagger \prime \prime}|0\rangle$. We would now like to decompose $\left|\psi^{i \prime \prime}\right\rangle$ with respect to the occupation of a given orbital $e$. To this aim, let us perform an orbital basis transformation using a unitary matrix $W$ to a basis in which $\hat{c}_{1}^{\dagger} \equiv \hat{c}_{e}^{\dagger}$,

$$
\left(\begin{array}{c}
\hat{c}_{1}^{\dagger \prime \prime} \\
\vdots \\
\hat{c}_{N}^{\dagger \prime \prime} \\
\vdots
\end{array}\right)=\left(\begin{array}{cccc}
W_{11} & \cdots & W_{1 N} & \cdots \\
\vdots & \ddots & \vdots & \\
W_{N 1} & \cdots & W_{N N} & \\
\vdots & & & \ddots
\end{array}\right)\left(\begin{array}{c}
\hat{c}_{1}^{\dagger} \\
\vdots \\
\hat{c}_{N}^{\dagger} \\
\vdots
\end{array}\right) .
$$

We can always decompose the upper $N \times N$ block of $W$ into a unitary matrix $\tilde{U}$ multiplied from the right with an upper triangular matrix $\tilde{V}$ with non-negative diagonal elements $0 \leqslant \tilde{V}_{i i} \leqslant 1$. By extending $\tilde{U}$ with the identity matrix for the remaining orbitals we can define the unitary matrix $U$ that gives

$$
\begin{aligned}
& \left(\begin{array}{cccc}
W_{11} & \cdots & W_{1 N} & \cdots \\
\vdots & \ddots & \vdots & \\
W_{N 1} & \cdots & W_{N N} & \\
\vdots & & & \ddots
\end{array}\right) \\
& =\left(\begin{array}{cccc}
\tilde{U}_{11} & \cdots & \tilde{U}_{1 N} & 0 \\
\vdots & \ddots & \vdots & 0 \\
\tilde{U}_{N 1} & \cdots & \tilde{U}_{N N} & 0 \\
0 & 0 & 0 & \mathbf{1}
\end{array}\right) \\
& \times\left(\begin{array}{cccc}
\tilde{V}_{11} & \cdots & \tilde{V}_{1 N} & \cdots \\
0 & \ddots & \vdots & \\
0 & 0 & \tilde{V}_{N N} & \cdots \\
W_{(N+1) 1} & \cdots & W_{(N+1) N} \cdots &
\end{array}\right) .
\end{aligned}
$$

The full $V$ matrix, i.e., $V=U^{\dagger} W$, corresponds to a valid orbital basis transformation since both $U$ and $W$ are unitary. Let us therefore consider the Slater determinant given by $\left|\psi^{i \prime}\right\rangle=\hat{c}_{1}^{\dagger \prime} \hat{c}_{2}^{\dagger \prime} \cdots \hat{c}_{N}^{\dagger \prime}|0\rangle$ where

$$
\hat{c}_{i}^{\dagger \prime}=\sum_{j} V_{i j} \hat{c}_{j}^{\dagger} .
$$

Since $V_{i 1}=0$ for $i=2,3, \ldots, N$ only $\hat{c}_{1}^{\dagger \prime}$ contributes to the occupation of $\hat{c}_{1}^{\dagger}=\hat{c}_{e}^{\dagger}$ in $\left|\psi^{i \prime}\right\rangle$. Furthermore, the operator $\sqrt{1-V_{11}} \hat{c}_{s} \equiv \sum_{j=2} V_{1 j} \hat{c}_{j}^{\dagger}$ fulfills $\left\{\hat{c}_{s}^{\dagger \prime}, \hat{c}_{i}^{\prime}\right\}=0$ for $i=$
$2,3, \ldots, N$ since $\left\{\hat{c}_{1}^{\dagger \prime}, \hat{c}_{i}^{\prime}\right\}=0$ for $i=2,3, \ldots, N$. We can therefore write [59]

$$
\left|\psi^{i \prime}\right\rangle=\left(\alpha^{\prime} \hat{c}_{e}^{\dagger}+\sqrt{1-\alpha^{\prime}} \hat{c}_{s}^{\dagger}\right) \hat{c}_{2}^{\dagger \prime} \cdots \hat{c}_{N}^{\dagger \prime}|0\rangle
$$

where $\alpha^{\prime}=V_{11}$. The Fock state $\left|\psi^{i \prime}\right\rangle$ is, however, identical to $\left|\psi^{i \prime \prime}\right\rangle$ since

$$
\begin{aligned}
\left|\psi^{i \prime}\right\rangle & =\hat{c}_{1}^{\dagger \prime} \hat{c}_{2}^{\dagger \prime} \cdots \hat{c}_{N}^{\dagger \prime}|0\rangle \\
& =\left(\sum_{j=1}^{N} U_{j 1}^{*} \hat{c}_{j}^{\dagger \prime \prime}\right)\left(\sum_{j=1}^{N} U_{j 2}^{*} \hat{c}_{j}^{\dagger \prime \prime}\right) \cdots\left(\sum_{j=1}^{N} U_{j N}^{*} \hat{c}_{j}^{\dagger \prime \prime}\right)|0\rangle \\
& =\operatorname{det} U \hat{c}_{1}^{\dagger \prime \prime} \hat{c}_{2}^{\dagger \prime \prime} \cdots \hat{c}_{N}^{\dagger \prime \prime}|0\rangle \\
& =\left|\psi^{i \prime \prime}\right\rangle .
\end{aligned}
$$

This implies that an arbitrary Fock state can always be decomposed as in Eq. (D20) with respect to the occupation of any single orbital $e$.

Let us now consider the squared overlap between the Fock state $\left|\psi^{i \prime}\right\rangle$ and the input state

$$
\left|\psi^{i}\right\rangle=\sqrt{\alpha} \hat{c}_{e}^{\dagger}\left|\psi_{e}\right\rangle+\sqrt{1-\alpha}\left|\psi_{s}\right\rangle
$$

The decomposition of $\left|\psi^{i \prime}\right\rangle$ in Eq. (D20) gives

$$
\begin{aligned}
\left|\left\langle\psi^{i \prime}\right\rangle \psi^{i}\right|^{2}= & \mid \sqrt{\alpha \alpha^{\prime}}\left\langle\psi_{e}^{\prime}\right\rangle \psi_{e} \\
& +\left.\sqrt{(1-\alpha)\left(1-\alpha^{\prime}\right)}\left\langle\psi_{e}^{\prime}\left|\hat{c}_{s}\right| \psi_{s}\right\rangle\right|^{2},
\end{aligned}
$$

where $\left|\psi_{e}^{\prime}\right\rangle=\hat{c}_{2}^{\dagger \prime} \cdots \hat{c}_{N}^{\dagger \prime}|0\rangle$. The overlap is maximized when the phase of $\hat{c}_{s}$ and the size of $\alpha^{\prime}$ are adjusted so that

$$
\begin{gathered}
\frac{\left\langle\psi_{e}^{\prime}\left|\hat{c}_{s}\right| \psi_{s}\right\rangle}{\left|\left\langle\psi_{e}^{\prime}\left|\hat{c}_{s}\right| \psi_{s}\right\rangle\right|}=\frac{\left\langle\psi_{e}^{\prime}\right\rangle \psi_{e}}{\left|\left\langle\psi_{e}^{\prime}\right\rangle \psi_{e}\right|}, \\
\alpha^{\prime}=\frac{\alpha\left|\left\langle\psi_{e}^{\prime}\right\rangle \psi_{e}\right|^{2}}{\alpha\left|\left\langle\psi_{e}^{\prime}\right\rangle \psi_{e}\right|^{2}+(1-\alpha)\left|\left\langle\psi_{e}^{\prime}\left|\hat{c}_{s}\right| \psi_{s}\right\rangle\right|^{2}},
\end{gathered}
$$

which yields

$$
\begin{aligned}
& \max _{\mid \psi^{\left.i^{\prime}\right\rangle}}\left|\left\langle\psi^{i \prime}\right\rangle \psi^{i}\right|^{2} \\
& \quad=\max _{\left|\psi_{e}^{\prime}\right\rangle, \hat{c}_{s}}\left[\alpha\left|\left\langle\psi_{e}^{\prime}\right\rangle \psi_{e}\right|^{2}+(1-\alpha)\left|\left\langle\psi_{e}^{\prime}\left|\hat{c}_{s}\right| \psi_{s}\right\rangle\right|^{2}\right] \\
& \quad \leqslant \alpha \max _{\left|\psi_{e}^{\prime}\right\rangle}\left|\left\langle\psi_{e}^{\prime}\right\rangle \psi_{e}\right|^{2}+(1-\alpha) \max _{\left|\psi_{s}^{\prime}\right\rangle}\left|\left\langle\psi_{s}^{\prime}|| \psi_{s}\right\rangle\right|^{2},
\end{aligned}
$$

where the maximization is over all normalized Fock states. The inequality in Eq. (D26) gives

$$
\begin{aligned}
1-\max _{\left|\psi^{i}\right\rangle}\left|\left\langle\psi^{i \prime}\right\rangle \psi^{i}\right|^{2} \geqslant & \alpha\left(1-\max _{\left|\psi_{e}^{\prime}\right\rangle}\left|\left\langle\psi_{e}^{\prime}\right\rangle \psi_{e}\right|^{2}\right) \\
& +(1-\alpha)\left(1-\max _{\left|\psi_{s}^{\prime}\right\rangle}\left|\left\langle\psi_{s}^{\prime}|| \psi_{s}\right\rangle\right|^{2}\right),
\end{aligned}
$$

which proves Eq. (D2) for the geometric particle entanglement measure. 
[1] R. Horodecki, P. Horodecki, M. Horodecki, and K. Horodecki, Quantum entanglement, Rev. Mod. Phys. 81, 865 (2009).

[2] T. E. Northup and R. Blatt, Quantum information transfer using photons, Nat. Photon. 8, 356 (2014).

[3] J. Volz, M. Weber, D. Schlenk, W. Rosenfeld, J. Vrana, K. Saucke, C. Kurtsiefer, and H. Weinfurter, Observation of Entanglement of a Single Photon with a Trapped Atom, Phys. Rev. Lett. 96, 030404 (2006).

[4] D. Leibfried, R. Blatt, C. Monroe, and D. Wineland, Quantum dynamics of single trapped ions, Rev. Mod. Phys. 75, 281 (2003).

[5] H. Häffner, C. Roos, and R. Blatt, Quantum computing with trapped ions, Phys. Rep. 469, 155 (2008).

[6] M. A. Eriksson, M. Friesen, S. N. Coppersmith, R. Joynt, L. J. Klein, K. Slinker, C. Tahan, P. M. Mooney, J. O. Chu, and S. J. Koester, Spin-based quantum dot quantum computing in silicon, Quant. Inf. Process. 3, 133 (2004).

[7] J. R. Schaibley, A. P. Burgers, G. A. McCracken, L.-M. Duan, P. R. Berman, D. G. Steel, A. S. Bracker, D. Gammon, and L. J. Sham, Demonstration of Quantum Entanglement Between a Single Electron Spin Confined to an Inas Quantum Dot and a Photon, Phys. Rev. Lett. 110, 167401 (2013).

[8] P. Zanardi, Quantum entanglement in fermionic lattices, Phys. Rev. A 65, 042101 (2002).

[9] H. Barnum, E. Knill, G. Ortiz, R. Somma, and L. Viola, A Subsystem-Independent Generalization of Entanglement, Phys. Rev. Lett. 92, 107902 (2004).

[10] A. P. Balachandran, T. R. Govindarajan, A. R. de Queiroz, and A. F. Reyes-Lega, Entanglement and Particle Identity: A Unifying Approach, Phys. Rev. Lett. 110, 080503 (2013).

[11] F. Benatti, R. Floreanini, and U. Marzolino, Sub-shot-noise quantum metrology with entangled identical particles, Ann. Phys. 325, 924 (2010).

[12] D. Dasenbrook, J. Bowles, J. B. Brask, P. P. Hofer, C. Flindt, and N. Brunner, Single-electron entanglement and nonlocality, New J. Phys. 18, 043036 (2016).

[13] N. Friis, A. R. Lee, and D. E. Bruschi, Fermionic-mode entanglement in quantum information, Phys. Rev. A 87, 022338 (2013).

[14] H. M. Wiseman and J. A. Vaccaro, Entanglement of Indistinguishable Particles Shared Between Two Parties, Phys. Rev. Lett. 91, 097902 (2003).

[15] M.-C. Bañuls, J. I. Cirac, and M. M. Wolf, Entanglement in fermionic systems, Phys. Rev. A 76, 022311 (2007).

[16] U. Marzolino and A. Buchleitner, Quantum teleportation with identical particles, Phys. Rev. A 91, 032316 (2015).

[17] L. Amico, R. Fazio, A. Osterloh, and V. Vedral, Entanglement in many-body systems, Rev. Mod. Phys. 80, 517 (2008).

[18] L. Ding, S. Mardazad, S. Das, S. Szalay, U. Schollwöck, Z. Zimborás, and C. Schilling, Concept of orbital entanglement and correlation in quantum chemistry, J. Chem. Theory Comput. 17, 79 (2021).

[19] J. Schliemann, J. I. Cirac, M. Kuś, M. Lewenstein, and D. Loss, Quantum correlations in two-fermion systems, Phys. Rev. A 64, 022303 (2001).

[20] K. Eckert, J. Schliemann, D. Bruß, and M. Lewenstein, Quantum correlations in systems of indistinguishable particles, Ann. Phys. 299, 88 (2002).

[21] Y. S. Li, B. Zeng, X. S. Liu, and G. L. Long, Entanglement in a two-identical-particle system, Phys. Rev. A 64, 054302 (2001).
[22] G. Ghirardi, L. Marinatto, and T. Weber, Entanglement and properties of composite quantum systems: A conceptual and mathematical analysis, J. Stat. Phys. 108, 49 (2002).

[23] G. C. Ghirardi and L. Marinatto, General criterion for the entanglement of two indistinguishable particles, Phys. Rev. A 70, 012109 (2004).

[24] G. C. Ghirardi and L. Marinatto, Identical particles and entanglement, Opt. Spectrosc. 99, 386 (2005).

[25] G. M. D'Ariano, F. Manessi, P. Perinotti, and A. Tosini, The feynman problem and fermionic entanglement: Fermionic theory versus qubit theory, Int. J. Mod. Phys. A 29, 1430025 (2014).

[26] N. Gigena and R. Rossignoli, Bipartite entanglement in fermion systems, Phys. Rev. A 95, 062320 (2017).

[27] T. Debarba, F. Iemini, G. Giedke, and N. Friis, Teleporting quantum information encoded in fermionic modes, Phys. Rev. A 101, 052326 (2020).

[28] E. Olofsson, P. Samuelsson, N. Brunner, and P. P. Potts, Quantum teleportation of single-electron states, Phys. Rev. B 101, 195403 (2020).

[29] C. W. J. Beenakker and M. Kindermann, Quantum Teleportation by Particle-Hole Annihilation in the Fermi Sea, Phys. Rev. Lett. 92, 056801 (2004).

[30] O. Sauret, D. Feinberg, and T. Martin, Electron spin teleportation current through a quantum dot array operating in the stationary regime, Phys. Rev. B 69, 035332 (2004).

[31] V. I. Anisimov, A. I. Poteryaev, M. A. Korotin, A. O. Anokhin, and G. Kotliar, First-principles calculations of the electronic structure and spectra of strongly correlated systems: Dynamical mean-field theory, J. Phys.: Condens. Matter 9, 7359 (1997).

[32] A. I. Lichtenstein and M. I. Katsnelson, Ab initio calculations of quasiparticle band structure in correlated systems: LDA++ approach, Phys. Rev. B 57, 6884 (1998).

[33] K. Held, Electronic structure calculations using dynamical mean field theory, Adv. Phys. 56, 829 (2007).

[34] V. Vedral, M. B. Plenio, M. A. Rippin, and P. L. Knight, Quantifying Entanglement, Phys. Rev. Lett. 78, 2275 (1997).

[35] V. B. Braginsky, Y. I. Vorontsov, and K. S. Thorne, Quantum nondemolition measurements, Science 209, 547 (1980).

[36] G. Nogues, A. Rauschenbeutel, S. Osnaghi, M. Brune, J. M. Raimond, and S. Haroche, Seeing a single photon without destroying it, Nature (Lond.) 400, 239 (1999).

[37] H.-W. Lee and J. Kim, Quantum teleportation and bell's inequality using single-particle entanglement, Phys. Rev. A 63, 012305 (2000).

[38] S. Yadav, Z. Zhu, and C. V. Singh, Defect engineering of graphene for effective hydrogen storage, Int. J. Hydr. Energy 39, 4981 (2014).

[39] L. Vaidman and N. Yoran, Methods for reliable teleportation, Phys. Rev. A 59, 116 (1999).

[40] L. Childress and R. Hanson, Diamond nv centers for quantum computing and quantum networks, MRS Bull. 38, 134 (2013).

[41] J. R. Weber, W. F. Koehl, J. B. Varley, A. Janotti, B. B. Buckley, C. G. Van de Walle, and D. D. Awschalom, Quantum computing with defects, Proc. Natl. Acad. Sci. USA 107, 8513 (2010).

[42] J. Wrachtrup, S. Y. Kilin, and A. P. Nizovtsev, Quantum computation using the $13 \mathrm{c}$ nuclear spins near the single nv defect center in diamond, Opt. Spectrosc. 91, 429 (2001).

[43] T. Astner, J. Gugler, A. Angerer, S. Wald, S. Putz, N. J. Mauser, 
M. Trupke, H. Sumiya, S. Onoda, J. Isoya, J. Schmiedmayer, P. Mohn, and J. Majer, Solid-state electron spin lifetime limited by phononic vacuum modes, Nat. Mater. 17, 313 (2018).

[44] G.-Q. Liu, X. Feng, N. Wang, Q. Li, and R.-B. Liu, Coherent quantum control of nitrogen-vacancy center spins near 1000 kelvin, Nat. Commun. 10, 1344 (2019).

[45] W. Pfaff, B. J. Hensen, H. Bernien, S. B. van Dam, M. S. Blok, T. H. Taminiau, M. J. Tiggelman, R. N. Schouten, M. Markham, D. J. Twitchen, and R. Hanson, Unconditional quantum teleportation between distant solid-state quantum bits, Science $\mathbf{3 4 5}$, 532 (2014).

[46] A. Gali, Theory of the neutral nitrogen-vacancy center in diamond and its application to the realization of a qubit, Phys. Rev. B 79, 235210 (2009).

[47] A. Ranjbar, M. Babamoradi, M. Heidari Saani, M. A. Vesaghi, K. Esfarjani, and Y. Kawazoe, Many-electron states of nitrogenvacancy centers in diamond and spin density calculations, Phys. Rev. B 84, 165212 (2011).

[48] P. Ji, R. Balili, J. Beaumariage, S. Mukherjee, D. Snoke, and M. V. Gurudev Dutt, Multiple-photon excitation of nitrogen vacancy centers in diamond, Phys. Rev. B 97, 134112 (2018).

[49] D. Loss and D. P. DiVincenzo, Quantum computation with quantum dots, Phys. Rev. A 57, 120 (1998).

[50] A. Imamoglu, D. D. Awschalom, G. Burkard, D. P. DiVincenzo, D. Loss, M. Sherwin, and A. Small, Quantum Information Processing Using Quantum Dot Spins and Cavity Qed, Phys. Rev. Lett. 83, 4204 (1999).

[51] D. P. DiVincenzo, D. Bacon, J. Kempe, G. Burkard, and K. B. Whaley, Universal quantum computation with the exchange interaction, Nature (Lond.) 408, 339 (2000).

[52] Z. Shi, C. B. Simmons, J. R. Prance, J. K. Gamble, T. S. Koh, Y.-P. Shim, X. Hu, D. E. Savage, M. G. Lagally, M. A. Eriksson,
M. Friesen, and S. N. Coppersmith, Fast Hybrid Silicon DoubleQuantum-Dot Qubit, Phys. Rev. Lett. 108, 140503 (2012).

[53] R. Sánchez, G. Granger, L. Gaudreau, A. Kam, M. PioroLadrière, S. A. Studenikin, P. Zawadzki, A. S. Sachrajda, and G. Platero, Long-Range Spin Transfer in Triple Quantum Dots, Phys. Rev. Lett. 112, 176803 (2014).

[54] F. de Pasquale, G. Giorgi, and S. Paganelli, Teleportation on a Quantum Dot Array, Phys. Rev. Lett. 93, 120502 (2004).

[55] R. L. de Visser and M. Blaauboer, Deterministic Teleportation of Electrons in a Quantum Dot Nanostructure, Phys. Rev. Lett. 96, 246801 (2006).

[56] H. Qiao, Y. P. Kandel, S. K. Manikandan, A. N. Jordan, S. Fallahi, G. C. Gardner, M. J. Manfra, and J. M. Nichol, Conditional teleportation of quantum-dot spin states, Nat. Commun. 11, 3022 (2020).

[57] P.-A. Mortemousque, E. Chanrion, B. Jadot, H. Flentje, A Ludwig, A. D. Wieck, M. Urdampilleta, C. Bäuerle, and T. Meunier, Coherent control of individual electron spins in a two-dimensional quantum dot array, Nat. Nanotechnol. 16, 296 (2021).

[58] We will not consider antiparticles (positrons) in this study which implies that any state vector must have a fixed number of electrons.

[59] C. V. Kraus, M. M. Wolf, J. I. Cirac, and G. Giedke, Pairing in fermionic systems: A quantum-information perspective, Phys. Rev. A 79, 012306 (2009).

[60] P. Thunström, I. Di Marco, and O. Eriksson, Electronic Entanglement in Late Transition Metal Oxides, Phys. Rev. Lett. 109, 186401 (2012).

[61] J. M. Zhang and M. Kollar, Optimal multiconfiguration approximation of an $n$-fermion wave function, Phys. Rev. A 89 , 012504 (2014). 